\title{
AAA+ protease-adaptor structures reveal altered conformations and ring specialization
}

\author{
Sora $\mathrm{Kim}^{1,3}$, Xue Fei ${ }^{1-3}$, Robert T. Sauer ${ }^{1}$, and Tania A. Baker ${ }^{1,4^{*}}$ \\ ${ }^{1}$ Department of Biology, Massachusetts Institute of Technology, Cambridge, MA 02139 \\ 2 Present address: Kymera Therapeutics Inc., Watertown, MA 02472 \\ 3 These authors contributed equally \\ ${ }^{4}$ Lead contact \\ * Correspondence: tabaker@mit.edu \\ ORCIDs: \\ Sora Kim: 0000-0002-9856-9574 \\ Xue Fei: 0000-0003-3378-7447 \\ Robert T. Sauer: 0000-0002-1719-5399 \\ Tania A. Baker: 0000-0002-0737-3411
}




\section{Summary}

CIpAP, a two-ring AAA+ protease, degrades $\mathrm{N}$-end-rule proteins bound by the ClpS adaptor. Here, we present high-resolution cryo-EM structures of ClpAPS complexes showing how CIpA pore loops interact with the ClpS N-terminal extension (NTE), which is normally intrinsically disordered. In two structural classes, the NTE is bound by a spiral of pore-1 and pore-2 loops in a manner similar to substrate-polypeptide binding by many AAA+ unfoldases. Kinetic studies reveal that pore-2 loops of the CIpA D1 ring catalyze protein remodeling required for substrate delivery by CIpS. In a third class, D2 pore-1 loops are rotated and tucked away from the channel, and do not bind the NTE, demonstrating asymmetry in engagement by the D1 and D2 rings. These studies demonstrate new structures and functions for key AAA+ elements. In addition to CIpAPS delivery, pore-loop tucking may be used broadly by $A A A+$ unfoldases, for example during enzyme pausing/unloading.

Keywords: ATPases associated with diverse cellular activities, cryo-EM, mechanism of adaptor mediated protein remodeling, pore-loop function, $\mathrm{N}$-end-rule degradation 


\section{INTRODUCTION}

Regulated proteolysis by energy-dependent AAA+ (ㅅTPases associated with diverse cellular activities) proteases maintains cellular homeostasis of proteins in all organisms. AAA+ proteases harness the energy of ATP hydrolysis to degrade regulatory proteins and proteins that are damaged, misfolded, or no longer needed (Sauer and Baker, 2011). AAA+ proteases, such as ClpAP, consist of a hexameric AAA+ unfoldase (e.g. $\left.\mathrm{ClpA}_{6}\right)$ and a self-compartmentalized peptidase (e.g. $\left.\mathrm{ClpP}_{14}\right)$. In the recognition step of degradation, a peptide sequence (degron) in a protein substrate is engaged by pore loops lining the axial channel of the AAA+ hexamer. Through conformational changes powered by ATP-hydrolysis cycles, native structure in the bound substrate is unfolded and processively translocated through the channel and into the peptidase chamber, where the polypeptide is cleaved into fragments. In addition to binding and engaging degrons directly, AAA+ proteases interact with adaptor proteins that modify their substrate specificity, often by tightening the affinity of the enzyme•substrate complex (Sauer and Baker, 2011; Olivares et al., 2015; Mahmoud and Chien, 2018).

Prokaryotes and eukaryotes use the $\mathrm{N}$-end-rule pathway to target proteins bearing specific $\mathrm{N}$-terminal residues (called N-degrons) for rapid degradation (Varshavsky, 2019). In Escherichia coli, the ClpS adaptor promotes CIpAP degradation of proteins containing Leu, Phe, Tyr, or Trp residues at the N-terminus (Tobias et al., 1991; Dougan et al., 2002; Zeth et al., 2002a, 2002b; Erbse et al., 2006; Varshavsky, 2019). The ClpS protein ( 10 kDa) docks with the N-terminal domain of ClpA and contains a hydrophobic pocket that binds the $\mathrm{N}$-end-rule residue (Zeth et al., 2002a, 2002b; Wang et al., 2008a; Román-Hernández et al., 2009). ClpS functions as a specificity switch for ClpAP, promoting degradation of $\mathrm{N}$-degron substrates while inhibiting degradation of ssrA-tagged and related substrates (Dougan et al., 2002; Guo et al., 2002; Erbse et al., 2006; Wang et al., 2008a). Unlike some adaptors that simply act as molecular matchmakers between the substrate and enzyme, ClpS also modulates the rate of ATP hydrolysis and substrate 
unfolding by ClpA. Interestingly, ClpS is proposed to interact with ClpA as a 'pseudo-substrate' (Dougan et al., 2002; De Donatis et al., 2010; Román-Hernández et al., 2011; Rivera-Rivera et al., 2014; Torres-Delgado et al., 2020). Specifically, the N-terminal extension (NTE) of free ClpS is exposed as an unstructured peptide, mimicking a degron. The NTE is poorly conserved among orthologs, with the exception of a short junction sequence adjacent to the ClpS core that typically contains a few tandem prolines (Hou et al., 2008; Román-Hernández et al., 2011). During ClpS-assisted degradation, a ClpS $\cdot N$-degron substrate complex initially binds to ClpA. Subsequently, the $\mathrm{N}$-degron substrate is transferred to ClpA for degradation and ClpS escapes destruction by mechanisms that are poorly understood.

Each ClpA subunit has two AAA+ modules, called D1 and D2, that associate in the hexamer to form two stacked rings (Grimaud et al., 1998). The D1 and D2 modules belong to different AAA+ subfamilies and have distinct biochemical functions (Erzberger and Berger, 2006). The D2 ring, a member of the HCLR AAA+ clade, is the principal ATPase motor responsible for unfolding and translocating substrates, including proteins with high thermodynamic stabilities (Kress et al., 2009; Kotamarthi et al., 2020; Zuromski et al., 2020). In contrast, the D1 ring, a classic AAA+ clade member, assists the D2 ring as an auxiliary motor, improves enzyme processivity, and plays a major role in substrate recognition (Kress et al., 2009; Kotamarthi et al., 2020; Zuromski et al., 2020, 2021). ClpS differentially regulates the activities of the D1 and D2 rings (Kress et al., 2009; Zuromski et al., 2021) via interactions of its NTE that we characterize here. Previous cryo-EM structures of ClpAP elucidate how the axial channel of the D1 and D2 rings engages the polypeptide of a directly recognized substrate (Lopez et al., 2020). Pore-1 and pore-2 loops in both rings form spiral-staircase-like arrangements that bind the substrate polypeptide, in a similar manner to those in structures of other double-ring AAA+ enzymes, such as Hsp104, ClpB, Cdc48/p97, and NSF (Zhao et al., 2016; Deville et al., 2017; Gates et al., 2017; White et al., 2018; Yu et al., 2018; Cooney et al., 2019; Lo et al., 2019; Rizo et al., 2019; Twomey et al., 2019). 
However, these previous structures do not provide insight into the distinct, specialized functions of each $\mathrm{AAA}+$ ring of ClpA or the mechanism of ClpS-assisted degradation of $\mathrm{N}$-degron substrates.

To characterize ring specialization and CIpS-CIpA collaboration, we solved cryo-EM structures of CIpAPS complexes that show how the normally disordered CIpS NTE assumes an extended conformation when bound in the CIpA axial channel. These structures reveal marked conformational differences from prior CIpAP structures (Lopez et al., 2020). We identify multiple conformations of ClpS-bound ClpA, including an arrangement in which the pore-1 loops of the D2 ring are tucked-in and face away from the channel, allowing only the D1 ring to interact strongly with the CIpS NTE. Mutagenesis and biochemical experiments establish that the pore-2 loops of the CIpA D1 ring are essential for ClpS delivery of an N-degron substrate but contribute little to docking of ClpS with ClpA. Our results demonstrate structural and functional plasticity among the ClpA pore loops, provide a structural basis for the functions of ClpS during N-degron substrate degradation, and contribute more broadly to understanding the operational modes available to $A A A+$ enzymes as they perform diverse biological processes.

\section{RESULTS}

\section{Distinct conformations of CIPAPS delivery complexes}

We used size-exclusion chromatography in the presence of ATP $\gamma \mathrm{S}$ to purify a complex of ClpA, ClpP, CIpS, and the N-end-rule substrate YLFVQELA-GFP (Figure 1A-B). Based on SDS-PAGE, the YLFVQELA-GFP substrate appeared to be sub-stoichiometric compared to ClpS (Figure 1B). Because ATP $\gamma$ S does not support degradation (Thompson et al., 1994; Hoskins et al., 1998; Ishikawa et al., 2001; Effantin et al., 2010; Miller and Lucius, 2014; Lopez et al., 2020), these complexes should represent early stages in ClpS-mediated delivery of $\mathrm{N}$-degron substrates. 
Following single-particle cryo-EM analyses (Figure S1), three-dimensional (3D) classification and reconstruction using RELION-3 yielded six density maps (3.22-3.38 $\AA$ ), representing three general structural classes (I, II, and III) with the latter classes being subdivided into $\mathrm{II}_{\mathrm{a}} / \mathrm{II}_{\mathrm{b}} / \mathrm{II}_{\mathrm{c}}$ or $\mathrm{III}_{\mathrm{a}} / \mathrm{III}$ bubclasses (Table 1; Figure S2). In low-pass filtered maps, the ClpS core domain (res. 27-106) could be docked into each of the six maps (Figure S3). In unfiltered maps, there was good density for all or part of the NTE of ClpS, for the D1 and D2 rings of CIpA, and for ClpP (Figure 1C). There was no substantial density for the core domain of ClpS, the N-domains of CIpA, or YLFVQELA-GFP, suggesting that these domains/proteins are not present in fixed conformations relative to the remaining parts of the complex or are potentially absent (YLFVQELA-GFP).

In our structures, the six subunits of the ClpA hexamer, which we label A through $F$ (clockwise direction with subunit $\mathrm{F}$ at the bottom), formed a shallow spiral, as expected from prior cryo-EM structures (Lopez et al., 2020). Six flexible IGL loops (res. 610-628) in each ClpA hexamer docked into clefts in the heptameric ring of ClpP, leaving one empty cleft (Figure 1D). Differences between classes I, II, and III include the relative positions of subunits in the CIpA spiral, density for the CIpS NTE in the ClpA channel, and changes within individual ClpA subunits (Figure 1EF; Figure 2). For example, density for the CIpS NTE was present in both the D1 and D2 rings of CIpA in classes I and II, but only in the D1 ring of class III (Figure 1F). In classes II and III, the relative height of ClpA subunits in the spiral was $A$ (highest) $>B>C>D>E>F$ (lowest), whereas in class-I subunit B was higher than subunit A, resulting in a more planar D1 ring (Figure 2A-B). Additionally, in the D2 ring of class-III structures, the small AAA+ domain of subunit E swings outward, breaking the rigid-body interface with its large AAA+ domain neighbor from subunit $F$ (Figure 2C). By contrast, structures of ClpAP with RepA-GFP and ATP $\gamma$ S (Lopez et al., 2020) did not display this feature, suggesting that it arises as a consequence of ClpS binding. The subclasses $\left(\mathrm{II}_{\mathbf{a}} / \mathrm{II}_{\mathbf{b}} / \mathrm{II}_{\mathbf{c}}\right.$ or $\left.\mathrm{III}_{\mathbf{a}} / \mathrm{II}_{\mathbf{b}}\right)$ differed from each other largely in the detailed interactions between 
CIpA and the CIpS NTE, the visibility of individual NTE residues, and the nucleotide occupancy of each ATPase site (ATP $\gamma$ S, ADP, or empty) (Figure S4-S5).

\section{Binding of the CIpS NTE within the axial channel of CIpA}

Each of our structures contained clear main-chain and side-chain density corresponding to all or part of the CIpS NTE (res. 2-26) in the CIpA channel (Figure 3A). The register of this ClpS peptide is very similar in each structure, with the C-terminal portion of the NTE (Pro ${ }^{24}-$ Pro $^{25}-$ Ser $^{26}$ ) near the top of the ClpA channel, and the $\mathrm{N}$-terminal portion near the bottom of the channel in classes I and II. The ClpA channel is lined by the D1 KYYR pore-1 loops (res. 258-260) and pore-2 loops (res. 292-302) and by the D2 GYVG pore-1 loops (res. 539-542) and pore-2 loops (res. 526-531). Pore-1 loops of AAA+ unfoldases and protein-remodeling machines contain a key,

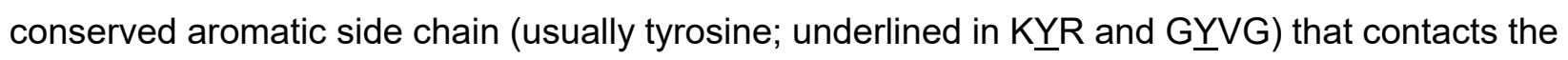
substrate polypeptide in the channel and functions in the binding, unfolding, and translocation of target proteins (Schlieker et al., 2004; Weibezahn et al., 2004; Hinnerwisch et al., 2005a; Martin et al., 2008; Doyle et al., 2012; losefson et al., 2015; Lopez et al., 2020; Zuromski et al., 2021).

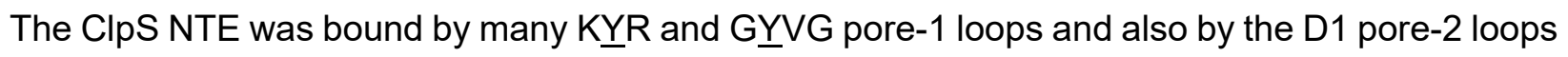
of ClpA. Neighboring pore-1 loops interacted with two-residue segments of the NTE, as observed for substrate polypeptides bound to multiple AAA+ unfoldases and protein-remodeling machines (Puchades et al., 2020).

Despite this overall resemblance to substrate engagement, there were deviations in individual pore-1 loop interactions from those in prior structures of ClpAP and some Hsp100 family members. For example, the D2 GYVG pore-1 loops of all six ClpA subunits contacted the ClpS NTE in classes $\mathbf{I}$ and $\mathbf{I I}_{\mathbf{c}}$ (Figure S5), whereas previous ClpAP structures and subclasses $\mathbf{I I}_{\mathbf{a}}$ and $I_{b}$ show four or five engaged GYYG loops (Figure S6B) (Lopez et al., 2020). The configuration of pore-1 loops in classes I and $\mathbf{I I}_{\mathbf{c}}$ was also different from an extended Hsp104•casein structure 
in which loops from both the top and bottom AAA+ rings of all six protomers contact substrate in a split 'lock-washer' conformation (Gates et al., 2017). In classes I and II , we observed five bound and one unbound pore-1 loops in D1 and six bound pore-1 loops in D2, an arrangement found in the high-affinity state of Mycobacterium tuberculosis ClpB (Yu et al., 2018). In many AAA+ structures, only the pore loops of ATP-bound subunits contact substrate (Puchades et al., 2020). By contrast and as reported for CIpAP.substrate complexes (Lopez et al., 2020), the pattern of engaged vs. disengaged pore loops in our structures did not strictly correlate with the nucleotide present in the corresponding ATPase active site (Figures S4-S5). For instance, ADP is bound to the class-II $\mathrm{D} 2$ nucleotide sites in subunits $\mathrm{E}$ and $\mathrm{F}$, but the GYVG loops from these domains contact the NTE. The presence of 11 engaged pore-1 loops (five D1 and six D2) likely contributes to the high-affinity of ClpAPS•N-degron complexes assembled in ATP $\gamma$ S (Román-Hernández et al., 2011).

\section{Pore-1 loops of D2 ring rotate out of the channel to alter polypeptide contacts}

In classes I and II, residues 2-15 of the CIpS NTE were built into density in the D2 portion of the channel, but this NTE region was not visible in class III, presumably as a consequence of its conformational heterogeneity. We infer that these NTE residues are within D2, as the more C-terminal NTE segment (res. 16-26) is bound by the D1 ring of class III in the same manner as in classes I and II. Thus, the two AAA+ rings of CIpA can differ in their engagement with the NTE, a feature not observed in substrate-bound CIpAP structures (Lopez et al., 2020).

This absence of density for the N-terminal portion of the NTE in class III correlated with distinct structural features within the axial channel. Most surprisingly, the D2 pore-1 loops in class III were rotated $\sim 90^{\circ}$ compared to their orientation in classes I and II, and the key $\mathrm{Tyr}^{540}$ side chains were tucked-in and turned away from the axial channel (Figure 3B-C; Movie S1). In both class-III subclasses, at least four of the six pore 1 loops were convincingly in this new tucked conformation. 
In many AAA+ unfoldases and protein-remodeling machines, one or two pore-1 loops, usually at the top and bottom of the spiral, are disengaged from the substrate polypeptide as a result of translational displacement of the corresponding subunit(s) (Deville et al., 2017; Gates et al., 2017; Puchades et al., 2017, 2019, 2020; Ripstein et al., 2017, 2020; Yu et al., 2018; Dong et al., 2019; Han et al., 2019, 2020; Lo et al., 2019; Fei et al., 2020a, 2020b; Lopez et al., 2020). This 'canonical' disengaged state of pore-1 loops in one or two subunits is very different than the tucked and rotated orientations of the class-III D2 pore-1 loops, in which no interactions with the polypeptide in the channel were present in the D2 ring. Pore-1 tyrosine contacts with the polypeptide within the AAA+ channel are considered essential for substrate binding and translocation. Thus, rotation of most (or all) $\mathrm{Tyr}^{540}$ side chains in the class-III D2 ring is sufficient to explain the lack of initial engagement of the N-terminal segment of the NTE and/or loss of binding that may occur during ClpS-assisted degradation of $\mathrm{N}$-degron substrates (see Discussion).

Three additional features of the class-III D2 ring are noteworthy. Coincident with the pore-1-loop rotation, the ClpA channel in the D2 ring of class III was wider than in classes I and II (Figure 2C). Second, as noted above, the D2 rigid-body interface between the small AAA+ domain of subunit $E$ and its neighboring large $A A A+$ domain in subunit $F$ was broken in class III. This rearrangement may facilitate the accompanying conformational changes that result in loss of NTE contacts by the D2 pore-1 loops. Finally, the D2 ring contained ADP in three adjacent subunits in class III, whereas classes I and II contained no more than two ADPs in the D2 ring (Figure S4). Thus, CIpA has the ability to bind all of a polypeptide in the axial channel tightly using pore loops in both rings or by disrupting coordinated activity of the D1 and D2 rings, to specifically bind only the C-terminal portion of this sequence within the D1 ring. 


\section{Pore-2 loops in the D1 ring form a second network of NTE-engaging contacts}

In addition to the pore-1 loop interactions described above, our structures show that at least four pore-2 loops (res. 292-302) in the D1 ring of ClpA contacted the ClpS NTE (Figure 4). In each subunit, these pore-2 contacts were positioned below the corresponding D1 KYYR contacts and were offset by $\sim 60^{\circ}$. The Ala ${ }^{295}-\mathrm{Ala}^{296}-\mathrm{Ser}^{297}$ tripeptide (AAS) at the tip of the D1 pore-2 loops contacted the opposing face of the CIpS NTE compared to the contacts made by the D1 pore-1 loops (compare orientation of D1 pore-2 loops on left vs. D1 pore-1 loops on right side of channel in Figure 4A). In contrast to the well-defined KYYR motif in the D1 pore-1 loop, which is conserved among Hsp104/CIpABC protein-remodeling enzymes and contains the invariant aromatic residue present in all $\mathrm{AAA}+$ unfoldases, the key residues and functions of the pore-2 loops have been poorly delineated to date (Puchades et al., 2020). Among ClpABC family members, the pore-2 loops are more variable in sequence and length (Figure 4B; Figure S6A).

To quantify the extent of pore-1 vs. pore-2 loop interactions in the D1 ring, we calculated the buried surface area (BSA) of the ClpS-NTE interface with each class of pore loops using PISA (Krissinel and Henrick, 2007). Mirroring the pattern of D1 pore-1 loops bound to the ClpS NTE, multiple pore-2 loops made significant CIpS NTE interactions in all class I, II, and III structures (Figure 4C). The D1 pore-2 loops made substantially larger contributions to the interface with the CIpS NTE than the pore-2 loops of the D2 ring, as the buried surface area contributed by the D1 pore-2 loops was comparable to those from either the D1 KYR or D2 GYVG pore-1 loops (Figure S6B). For example, in class $\mathbf{I I}_{\mathbf{c}}$, the BSA values for the D1 KYR loops range from 63 to $196 \AA^{2}$, the D1 pore-2 loops range from 40 to $156 \AA^{2}$, and D2 GYVG pore-1 loops range from 101 to 179 $\AA^{2}$. In contrast, the D2 pore-2 loops only weakly contacted the NTE, as BSA values of these interactions range from 20 to $74 \AA^{2}$. Thus, pore loops in the D1 ring make a greater total number of NTE interactions than pore loops in the D2 ring. The extensive network of NTE-engaging residues in the D1 ring suggests that it has more specific polypeptide binding/recognition 
'capacity' than the D2 ring, as predicted by biochemical studies (Hinnerwisch et al., 2005a; Zuromski et al., 2021).

\section{D1 pore-2 loops mediate substrate unfolding and mechanical remodeling of ClpS}

To test the functional importance of the D1 pore-2 loops, we mutated the AAS sequence (res. 295-297) to increase bulkiness (QTQ), to mimic the pore-1 loop (KYR), to increase flexibility (GGG), or to delete this tripeptide $(\Delta 295-297)$. As a defect in ClpS binding with these mutants was one reasonable hypothesis based on our structures, we first assayed assembly of ternary $\mathrm{ClpA}_{6} \cdot \mathrm{ClpS} \cdot \mathrm{N}$-degron peptide complexes using fluorescence anisotropy (Figure 5A). Strikingly, all pore-2 loop variants maintained tight affinity for the ClpS•N-degron complexes and behaved similarly to wild-type CIpA ( $\left.{ }^{\mathrm{WT}} \mathrm{ClpA}\right)$ in the control experiment that monitored the binary affinity of CIpA to the N-degron peptide. We then used these variants to assay ClpAPS degradation of the $\mathrm{N}$-degron substrate YLFVQELA-GFP (Figure 5B). Notably, all of the D1 pore-2-loop variants except QTQ were unable to degrade this substrate. These defects could arise from an inability to unfold or translocate YLFVQELA-GFP or from failure to transfer the YLFVQELA-GFP substrate from CIpS to CIpA.

For each variant, we then determined the ATP-hydrolysis rate of CIpA alone and in the presence of ClpP, ClpS, and/or a directly recognized protein substrate. The ATPase rate serves as an indirect readout of functional ClpA assembly with its binding partners, which differentially modulate ATP hydrolysis by ClpA. For instance, ClpP binding stimulates the ATPase rate of

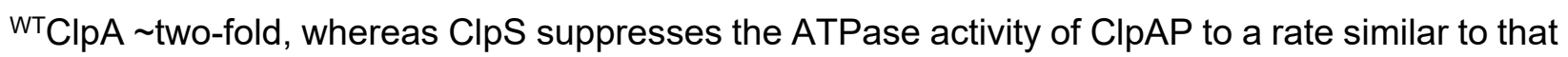
of ClpA alone (Hinnerwisch et al., 2005b; Hou et al., 2008). All of the pore-2 variants had basal ATPase rates comparable to ${ }^{\mathrm{WT}} \mathrm{ClpA}$ and exhibited ATPase modulation by ClpP and ClpS that was generally similar to wild-type (Figure S7A). Furthermore, in the presence of the super-folder

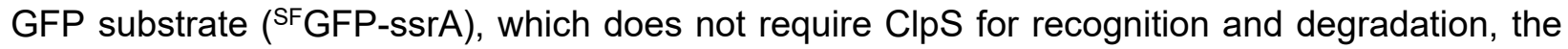


ATPase rate of each CIpAP variant (with the exception of ${ }^{\mathrm{KYR} C I p A P)}$ was moderately reduced during substrate processing, as expected from a previous study reporting $\sim 20 \%$ suppression of ATP hydrolysis by GFP-ssrA (Kress et al., 2009). We conclude based on these studies that our D1 pore-2 loop mutations do not grossly alter CIpA ATPase activity and are also unlikely to substantially change ClpA assembly with CIpP, ClpS, or SFGFP-ssrA.

Next, we assayed the ability of these D1 pore-2-loop variants to degrade FITC-casein, a molten-globule protein that does not require ClpS for recognition or robust CIpAP unfolding activity

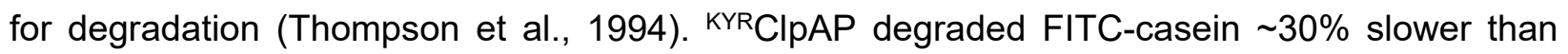

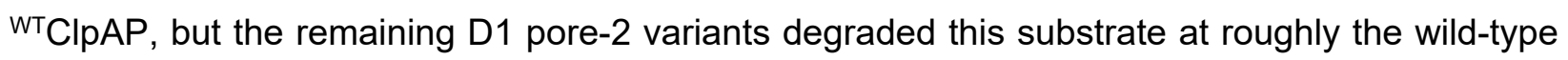
rate (Figure S7B), indicating that recognition and translocation of this substrate are not substantially affected by the D1 pore-2 loop mutations. We then assayed the effects of the ClpA D1 pore-2 loop mutations on the steady-state kinetics of SFGFP-ssrA degradation, a highly stable native substrate (Figure S7C). $K_{M}$ values for degradation of this substrate by ${ }^{{ }^{\top} \mathrm{ClpAP}}$ and the D1 pore-2 loop variants were within error, suggesting that the D1 pore-2 loops play little, if any, role in recognition of this substrate. $V_{\max }$ for SFGFP-ssrA degradation was unaffected by the QTQ mutation, reduced $\sim$ two-fold by the GGG and $\Delta 295-297$ mutations, and reduced $\sim$ six-fold for the KYR mutant. Based on these results, we conclude that the D1 pore-2 loops can promote, but are not essential for, a reaction step after initial substrate recognition, presumably GFP unfolding, which is rate limiting for degradation (Singh et al., 2000). Importantly however, these partial defects in unfolding by the GGG, $\Delta 295-297$, and KYR variants are insufficient to explain the complete inability of these mutants to degrade YLFVQELA-GFP when delivered by CIpS.

In comparison to FITC-casein and SFGFP-ssrA, which are directly recognized by ClpAP, degradation of ClpS-dependent substrates require an additional protein-remodeling step. That is, ClpA must remodel ClpS, to allow substrate transfer to ClpA, and then unfold the N-degron 
substrate. Concurrently, ClpS reduces the ClpA ATPase rate, which in turn slows unfolding and translocation (Dougan et al., 2002; Hou et al., 2008; De Donatis et al., 2010; Román-Hernández et al., 2011; Rivera-Rivera et al., 2014; Torres-Delgado et al., 2020). Therefore, ClpS should inhibit N-degron substrate degradation by the ClpA D1 pore-2 loop variants that we infer lack sufficient unfolding activity to remodel ClpS and transfer the substrate from ClpS to ClpA. We tested this hypothesis by measuring the degradation rates of YLFVQELA-GFP in the absence and presence of ClpS (Figure 5C). Although recognition of $\mathrm{N}$-end-rule substrates by ClpAP alone is intrinsically weak and normally enhanced by ClpS (Wang et al., 2007), the addition of ClpS hindered YLFVQELA-GFP degradation by the KYR, GGG, and $\Delta 295-297$ variants, but not by

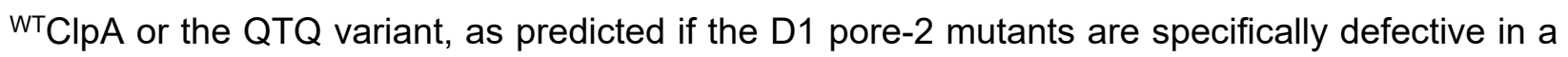
CIpS remodeling step required for efficient $\mathrm{N}$-degron substrate degradation.

In summary, these data suggest that the sequence identity of the AAS tripeptide (res. 295-297) alone is not critical for D1 pore-2 loop activity, as substituting these residues with QTQ had little effect on ATP hydrolysis and degradation of all substrates tested (Figure 5B-C; Figure S7). Instead, changing the chemical/conformational properties of this loop by altering charge/aromaticity (KYR) or flexibility (GGG and $\Delta 295-297$ ) had more profound effects. The severe defects in ClpAPS degradation conferred by the deleterious D1 pore-2 mutations but unchanged $\mathrm{ClpS} \cdot \mathrm{N}$-degron assembly support the conclusion that D1 pore-2 loops assist in mechanical work needed to transfer the $\mathrm{N}$-degron substrate from the adaptor to the protease (and perhaps also for subsequent reaction steps) but are not required for adaptor/substrate docking with CIpAP. 


\section{DISCUSSION}

\section{The CIpS NTE is a "degron mimic"}

Our ClpAP-ClpS structures, taken with previous CIpAP structures and those of additional AAA+ family members, illustrate the variety of functional conformations $A A A+$ unfoldases can adopt to perform their biological functions. Importantly in all our structures, interactions between the ClpS NTE and pore loops in the CIpA channel mimic contacts observed with a polypeptide segment of the protein substrate in prior ClpA structures (Lopez et al., 2020). Specifically, the conserved tyrosines from adjacent pore-1 loops in the D1 (KYRR) and D2 (GYG) ring contact every second residue of the NTE polypeptide (Figure S5), with additional contacts mediated by the pore-2 loops of the D1 ring. Thus, in addition to its interaction with the CIpA N-domain, ClpS uses its NTE to dock tightly with the CIpA channel during substrate delivery.

Our ClpAPS structures were assembled in ATP $\gamma$ S, which ClpA does not hydrolyze, demonstrating that binding of the entire CIpS NTE within both CIpA rings does not require hydrolysis-dependent power strokes. Together with biochemical studies and structures of substrate complexes with ATP $\gamma$ S-bound ClpA (Hoskins et al., 1998, 2000; Lopez et al., 2020), these results suggest that any polypeptide in an unfolded/misfolded protein could passively enter an open ClpA channel, enabling CIpAP to function broadly in general protein quality control. Indeed, most of the ClpS NTE sequence is poorly conserved among orthologs and can be changed without compromising delivery of $\mathrm{N}$-end-rule substrates (Hou et al., 2008), suggesting that ClpA can engage many different sequences. By contrast, the full axial channel of the ClpXP protease is blocked by a pore-2 loop prior to initiation of unfolding and translocation, probably limiting binding to proteins bearing highly specific ClpX degrons (Fei et al., 2020b).

Our structures also reveal that ClpA pore loops bind and engage the ClpS NTE, and thus can apply mechanical force to the ClpS core domain during the $\mathrm{N}$-degron delivery process. Previous 
studies demonstrate that the CIpS NTE enters the CIpA axial channel during assembly of delivery complexes and also can independently function as a degron for ClpAP, providing biochemical evidence that the CIpA pore loops can 'pull' on the NTE to remodel ClpS (Román-Hernández et al., 2011; Rivera-Rivera et al., 2014). Blocking the CIpS NTE from entering the channel inhibits ClpS-assisted substrate degradation, reinforcing the importance of ClpA 'pulling' on the CIpS NTE during N-degron delivery (Rivera-Rivera et al., 2014). The degron-like binding of the NTE provides a structural basis for the delivery mechanism depicted in Figure 6, in which CIpA pore loops engage the NTE and power strokes resulting from ATP hydrolysis transmit force to mechanically remodel ClpS and thereby promote transfer of the N-end-rule substrate from ClpS to ClpAP for degradation.

Despite the degron-like interactions of the NTE with CIpA, ClpS is not degraded (Dougan et al., 2002; Román-Hernández et al., 2011). Interestingly, mutation of Pro ${ }^{24}-$ Pro $^{25}$ to Ala ${ }^{24}-\mathrm{Ala}^{25}$ near the ClpS NTE-core junction generates a CIpS variant that can be degraded by CIpAP (RiveraRivera, 2015; Zuromski et al., 2021). In our structures, Pro ${ }^{24}-$ Pro $^{25}$ binds near the top of the CIpA channel, and the ClpS core domain is flexibly positioned directly above the pore. Although our structures only capture the initial docking of CIpS with CIpA, we propose that during subsequent stages of ClpS delivery, ClpA pore loops may not grip Pro ${ }^{24}-\mathrm{Pro}^{25}$ strongly enough to fully unfold CIpS, leading to 'back-slipping' in the channel and thus CIpS release. Such slipping is likely a consequence of the unique chemical properties of proline, which lacks an amide hydrogen and cannot form the extended peptide conformation adopted by the rest of the NTE in our structures and by substrate polypeptides in the channels of many other AAA+ unfoldases (Puchades et al., 2020). ClpXP translocates poly-proline at an even slower rate and with a higher cost of ATP hydrolysis cycles than poly-glycine, another homopolymer that leads to pore-loop 'slipping' (Barkow et al., 2009; Bell et al., 2019). Other types of 'slippery' sequences adjacent to folded domains have been shown to cause release of truncated degradation products by a number of 
AAA+ proteases (Lin and Ghosh, 1996; Levitskaya et al., 1997; Sharipo et al., 2001; Hoyt et al., 2006; Daskalogianni et al., 2008; Kraut et al., 2012; Kraut, 2013; Too et al., 2013; Vass and Chien, 2013; Bell et al., 2019). Partial ClpAP processing of native ClpS does not occur because its NTE is not long enough to enter the ClpP peptidase chamber (Figure 1F), but is observed for a variant bearing a duplicated NTE of $\sim 50$ residues (Rivera-Rivera et al., 2014).

\section{Implications of tucked pore-1 loops in the D2 ring}

In the D2 ring of our class-III structures, many GYYVG pore-1 loops assume a 'tucked' conformation in which they rotate away from the center of the CIpA axial channel and do not engage the ClpS NTE (Figure 3B-C), presumably weakening ClpS•ClpA binding. There are several functional implications. First, the D2-disengaged/D1-engaged species could represent an intermediate in the assembly of higher-affinity ClpAPS complexes in which both rings engage the NTE (Figure 6A). Second, tucked D2 GYVG pore loops could be important during latter steps in ClpS-dependent substrate delivery (Figure 6B), which require conformational remodeling of the ClpS core to weaken its interactions with the $\mathrm{N}$-degron substrate and promote its transfer to ClpA (Hou et al., 2008; Román-Hernández et al., 2011; Rivera-Rivera et al., 2014). For example, after failed attempts by CIpA to fully unfold the ClpS core, release of the NTE from the D2 ring could increase the probability that ClpS dissociates completely from CIpAP, freeing the D2 pore loops to engage the $\mathrm{N}$-degron substrate for degradation (see Figure 6D). Finally, pore-loop tucking does not require ATP hydrolysis, suggesting that under certain conditions (e.g., when bound to the CIpS NTE), ClpA readily adopts the class-III structures, which constitute $\sim 20 \%$ of particles in our final dataset.

More broadly, pore-loop tucking may be used during the process of enzyme pausing and/or unloading by CIpA and other AAA+ unfoldase motors. For example, the ClpA D1 ring functions as a 'back up' motor to prevent pausing when the principal D2-ring motor fails (Kotamarthi et al., 
2020; Zuromski et al., 2021). Transiently breaking contacts with the polypeptide via pore-loop tucking in only the D2 ring would allow the weaker D1 ring to continue unfolding/translocation without working against the stalled D2 motor. Subsequent 'untucking' of the D2 pore-1 loops once the sequence causing the pause is cleared would allow the D2 motor to re-engage, restarting robust translocation by both rings. More generally, concerted loss of peptide contacts by all AAA+ domains within a ring via pore-loop rotation and tucking would be an efficient mechanism for an unfoldase either to transiently disengage from a bound polypeptide or facilitate full enzyme dissociation upon failure of a AAA+ motor to unfold, translocate, or remodel a bound protein. By contrast, dissociation of a AAA+ enzyme from its polypeptide track by transitioning from a closed, substrate-bound right-handed spiral to an open, left-handed 'lock-washer' observed in some Hsp100 family members (Yokom et al., 2016; Gates et al., 2017; Yu et al., 2018) requires much larger, global conformational changes throughout the AAA+ hexamer.

\section{Specialized functions of pore-2 loops}

The pore-2 loops of other AAA+ unfoldases/remodeling enzymes have been shown to contact substrate polypeptides (Johjima et al., 2015; Deville et al., 2017; Gates et al., 2017; Puchades et al., 2017, 2019; Alfieri et al., 2018; Yu et al., 2018; Sandate et al., 2019; Zehr et al., 2020; Fei et al., 2020a, 2020b; Han et al., 2020; Shin et al., 2021; Kavalchuk et al., 2022). The AAS residues of the CIpA D1 pore-2 loops make substantial contacts with the CIpS NTE. Nevertheless, we find that these interactions are not critical for $\mathrm{ClpA} \cdot \mathrm{ClpS}$ binding but instead help mediate mechanical work needed during ClpS-assisted N-end-rule degradation (Figure 5; Figure 6B). We propose that the D1 pore-2 loops of ClpA collaborate with the D2 pore-1 loops, which are also required for ClpS delivery (Zuromski et al., 2021), in mechanical remodeling of ClpS and/or substrate transfer to CIpA. Both sets of loops could contribute to coordinated pulling on the NTE to apply force to and remodel ClpS. Next, the pore-2 loops could capture and initiate unfolding of the 'released' $\mathrm{N}$-degron substrate, and generate a sufficiently long polypeptide 'tail' to reach the more powerful 
D2 pore-1 loops (Figure 6C-D). Meanwhile, the D2 pore-1 loops could release the CIpS NTE via concerted loop-tucking, but then 'untuck' to grab this 'tail' for processive substrate unfolding and translocation. Future studies parsing the interaction of pore-1 and pore-2 loops in both ClpA rings are needed to further elucidate the mechanistic steps of $\mathrm{N}$-degron substrate delivery and degradation, as well as to understand why pore-2 loops are critical in CIpS-mediated degradation but less important for other classes of substrates.

\section{ClpA functions using both coordinated and independent action of the D1 and D2 rings}

Loss of D2 pore-1 loops engagement with the CIpS NTE is a major feature distinguishing our class-III structures from classes I and II. Although the asymmetric engagement of substrate in the D1 but not the D2 ring of class III has some parallels with substrate-bound structures of NSF and Pex1•Pex6 (Blok et al., 2015; Gardner et al., 2018; White et al., 2018), the substrate-binding rings of these other enzymes adopt a 'canonical' right-hand spiral organization, whereas the ring that does not bind substrate assumes a planar conformation. In contrast, the non-binding, ClpA D2 ring in class III remains in the right-handed spiral conformation. Moreover, the portion of the ClpS NTE in the D1 ring of class-III structures is bound in the same fashion as our class-I and class-II structures. This structural snapshot of 'divided' NTE engagement between the D1 and D2 pore-1 loops reinforces biophysical and biochemical experiments that reveal a division of labor between the two AAA+ modules of ClpA (Kress et al., 2009; Kotamarthi et al., 2020; Zuromski et al., 2021). Multiple studies of other double-ring remodeling/unfoldase enzymes, including ClpB, Hsp104, ClpC, Cdc48/p97/VCP, and the ribosomal assembly factor Rix7, report the separation of substrate binding/recognition functions in one ring from the role of the second ring as the principal motor performing mechanical work (Hattendorf and Lindquist, 2002; Mogk et al., 2003; Wang et al., 2011; Doyle et al., 2012; Bodnar and Rapoport, 2017a, 2017b; Lo et al., 2019). These results illustrate that functional specialization of individual rings is emerging as a theme shared by many double-ring $\mathrm{AAA}+$ unfoldases and protein-remodeling enzymes. 


\section{Author Contributions}

Conceptualization, S.K., X.F., R.T.S., and T.A.B.; Methodology, S.K., X.F., T.A.B., and R.T.S;

Validation, S.K., X.F., and R.T.S.; Formal Analysis, S.K. and X.F.; Investigation, S.K. and X.F.;

Resources, S.K.; Writing - Original Draft, S.K. and X.F.; Writing - Review \& Editing, S.K., X.F., R.T.S., and T.A.B.; Visualization, S.K. and X.F.; Supervision, R.T.S. and T.A.B.; Funding Acquisition, S.K., R.T.S., and T.A.B.

\section{Acknowledgments}

We thank E. Brignole and P. Dip for support in preparing and screening cryo-EM grids at the MIT.nano Automated Cryogenic Electric Microscopy Facility on a Talos Arctica microscope, which was a gift from the Arnold and Mabel Beckman Foundation, and C. Xu, K. Song, and K. Lee for data collection at the Cryo-EM Core Facility at the University of Massachusetts Chan Medical School. We thank I. Levchenko for advice preparing ClpAPS complexes and S. Bell, T. Bell, J. Park Morehouse, T. Shih, J. Zhang, and K. Zuromski for helpful advice and feedback.

This work was supported by NIH grant AI-016892 (R.T.S., T.A.B.), the Howard Hughes Medical Institute (T.A.B.), and the National Science Foundation Graduate Research Fellowship grant 1745302 (S.K.). The content is solely the responsibility of the authors and does not necessarily represent the official views of the National Institutes of Health.

\section{Declarations of Interest}

The authors declare no competing interests. 
Table 1. Cryo-EM data collection, processing, model building, and validation statistics.

\begin{tabular}{|c|c|c|c|c|c|c|}
\hline class name & A & \multicolumn{3}{|c|}{$\mathbf{B}$} & \multicolumn{2}{|c|}{$\mathbf{C}$} \\
\hline PDB ID & $\mathrm{XXX}$ & $\mathrm{XXX}$ & $\mathrm{XXX}$ & $\mathrm{XXX}$ & $\mathrm{XXX}$ & $\mathrm{XXX}$ \\
\hline EMDB ID & $\overline{X X X}$ & $\mathrm{XXX}$ & $\mathrm{XXX}$ & $\mathrm{XXX}$ & $\overline{X X X}$ & $\mathrm{XXX}$ \\
\hline \multicolumn{7}{|c|}{ data collection and processing } \\
\hline microscope & \multicolumn{6}{|c|}{ Talos Arctica } \\
\hline camera & \multicolumn{6}{|c|}{$\mathrm{K} 3$} \\
\hline magnification & \multicolumn{6}{|c|}{$45,000 x$} \\
\hline voltage $(\mathrm{kV})$ & \multicolumn{6}{|c|}{200} \\
\hline total electron dose $\left(\mathrm{e}^{-} / \AA^{2}\right)$ & \multicolumn{6}{|c|}{34.71} \\
\hline defocus range $(\mu \mathrm{m})$ & \multicolumn{6}{|c|}{-0.5 to -2.5} \\
\hline pixel size $(\AA)$ & \multicolumn{6}{|c|}{0.435} \\
\hline micrographs collected & \multicolumn{6}{|c|}{9169} \\
\hline initial particles & \multicolumn{6}{|c|}{$1,043,033$} \\
\hline final particles & 51,750 & 156,677 & 43,431 & 37,530 & 37,885 & 31,453 \\
\hline symmetry & C1 & C1 & C1 & C1 & C1 & C1 \\
\hline $\begin{array}{l}\text { map resolution }(\AA) \text { at } \\
0.143 \text { FSC threshold }\end{array}$ & 3.24 & 3.38 & 3.33 & 3.24 & 3.22 & 3.26 \\
\hline \multicolumn{7}{|c|}{ model composition } \\
\hline non-hydrogen atoms & 38021 & 37900 & 37776 & 38131 & 37880 & 38006 \\
\hline protein residues & 4820 & 4805 & 4792 & 4837 & 4819 & 4823 \\
\hline nucleotides & 12 & 11 & 10 & 12 & 12 & 12 \\
\hline \multicolumn{7}{|c|}{ refinement } \\
\hline map-model CC & 0.80 & 0.77 & 0.75 & 0.81 & 0.81 & 0.82 \\
\hline RMSD bond lengths $(\AA)$ & 0.005 & 0.005 & 0.005 & 0.005 & 0.006 & 0.006 \\
\hline RMSD bond angles $\left({ }^{\circ}\right)$ & 1.115 & 1.117 & 1.100 & 1.095 & 1.156 & 1.142 \\
\hline \multicolumn{7}{|c|}{ validation } \\
\hline MolProbity score & 1.09 & 1.06 & 1.06 & 1.07 & 1.14 & 1.10 \\
\hline clash score & 2.98 & 2.74 & 2.74 & 2.78 & 3.48 & 3.05 \\
\hline $\mathrm{C} \beta$ deviation $(\%)$ & 0.00 & 0.00 & 0.00 & 0.00 & 0.00 & 0.00 \\
\hline rotamer outliers (\%) & 0.00 & 0.00 & 0.02 & 0.00 & 0.00 & 0.00 \\
\hline $\begin{array}{c}\text { Ramachandran favored } \\
(\%)\end{array}$ & 99.94 & 99.73 & 99.75 & 99.88 & 99.77 & 99.50 \\
\hline $\begin{array}{l}\text { Ramachandran } \\
\text { disallowed }(\%)\end{array}$ & 0.00 & 0.00 & 0.00 & 0.00 & 0.00 & 0.00 \\
\hline
\end{tabular}




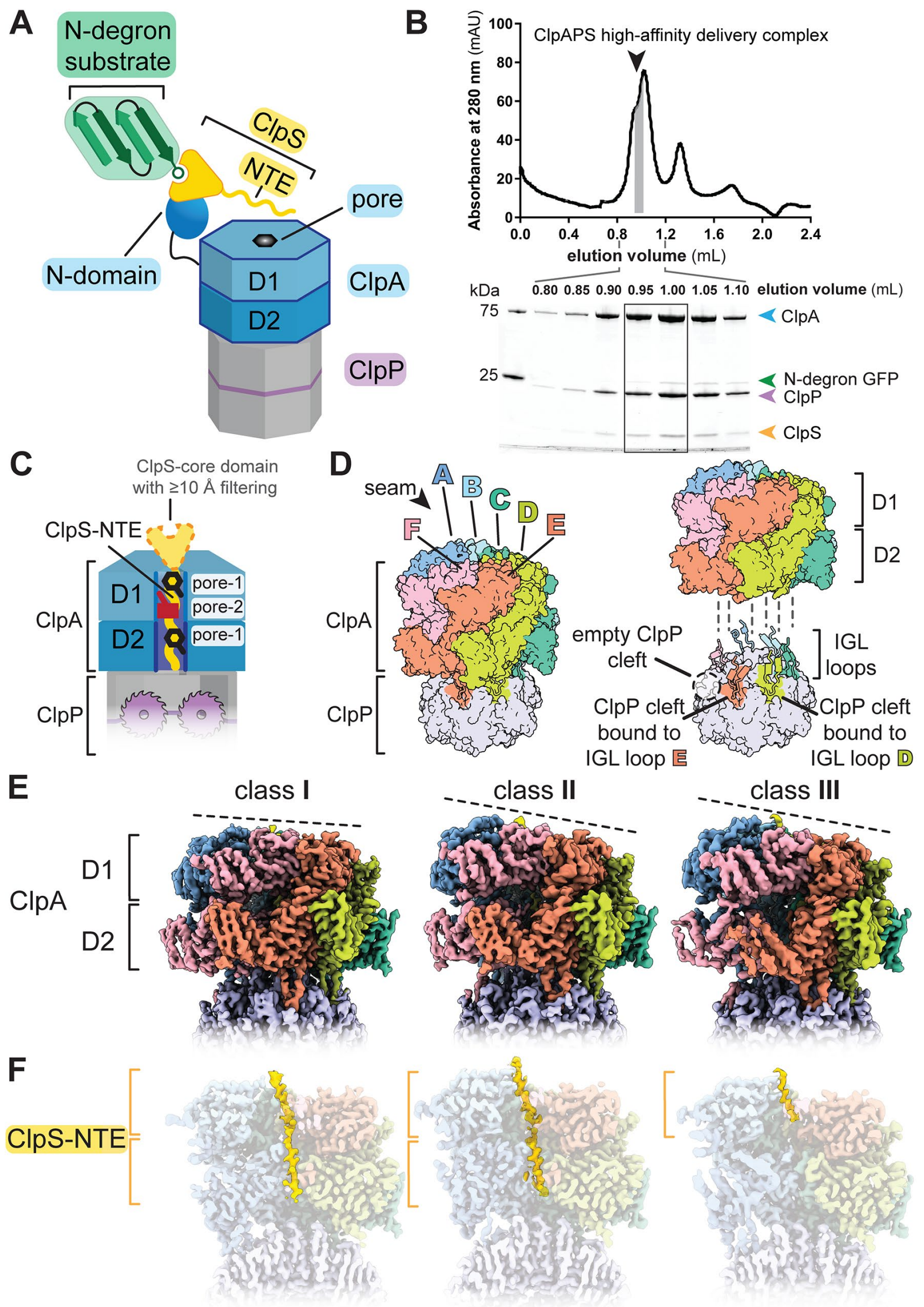


Figure 1. Architectures of ClpS-bound ClpAP

(A) Cartoon of ClpS delivery of an N-degron substrate for ClpAP degradation. Native ClpS (yellow wedge) binds to the $\mathrm{N}$-degron substrate (green) and also binds to an $\mathrm{N}$-terminal domain of ClpA (blue oval)

(B) Size-exclusion chromatography (top panel) of a complex of CIpA, CIpP, ClpS, and YLFVQELAGFP (an N-degron substrate) assayed by SDS-PAGE (bottom panel). Gray shaded area and boxed area indicate the fractions pooled for cryo-EM.

(C) Cartoon of pore loops that interact with the NTE and proteins resolved in cryo-EM structures. (D) (left panel) ClpA subunit nomenclature in right-hand spiral hexamer, where the seam interface is between the lowest $(F)$ subunit and the highest $(A)$ subunit. The ClpA hexamer docks into clefts in the $\mathrm{ClpP}_{7}$ ring via IGL loops (right panel). The empty ClpP cleft is located between the clefts occupied by subunits $E$ and $F$.

(E) Side views of the cryo-EM maps of classes $\mathbf{I}, \mathbf{I I}_{\mathbf{c}}$, and $\mathbf{I I I}_{\mathbf{b}}$. The dashed line indicates the relative height of ClpA subunits within the spiral.

(F) Cutaway views of panel E showing density for the CIpS NTE colored yellow. 
A

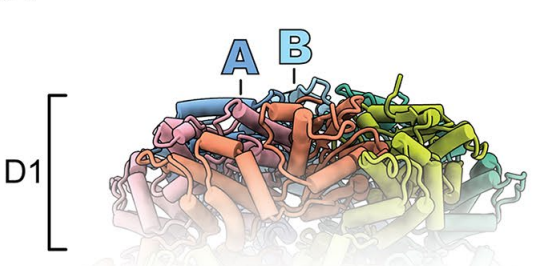

class II

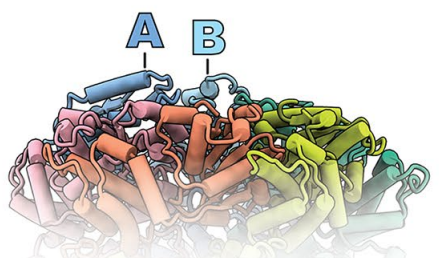

class III

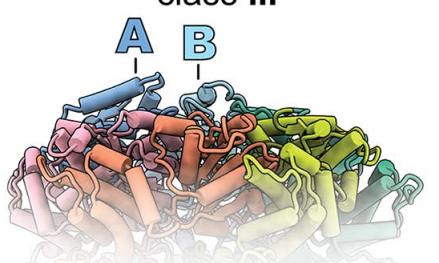

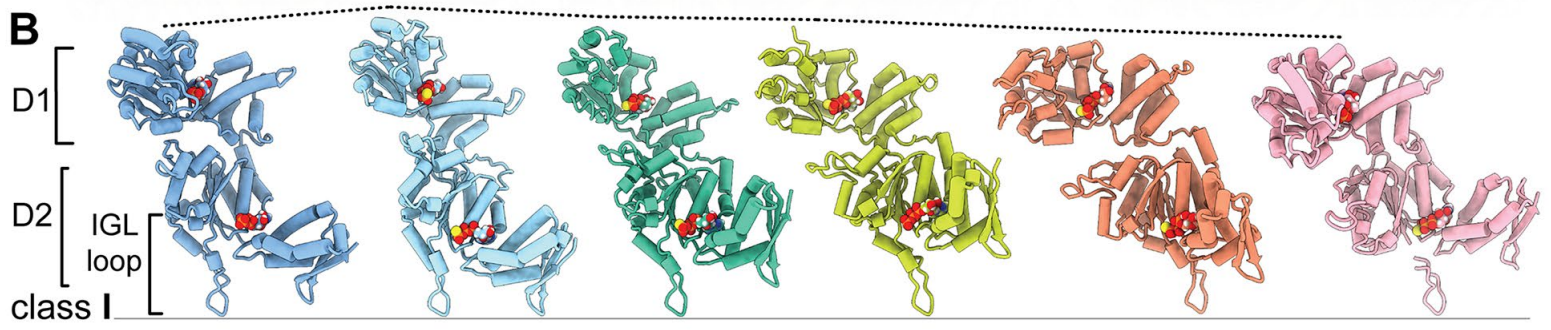
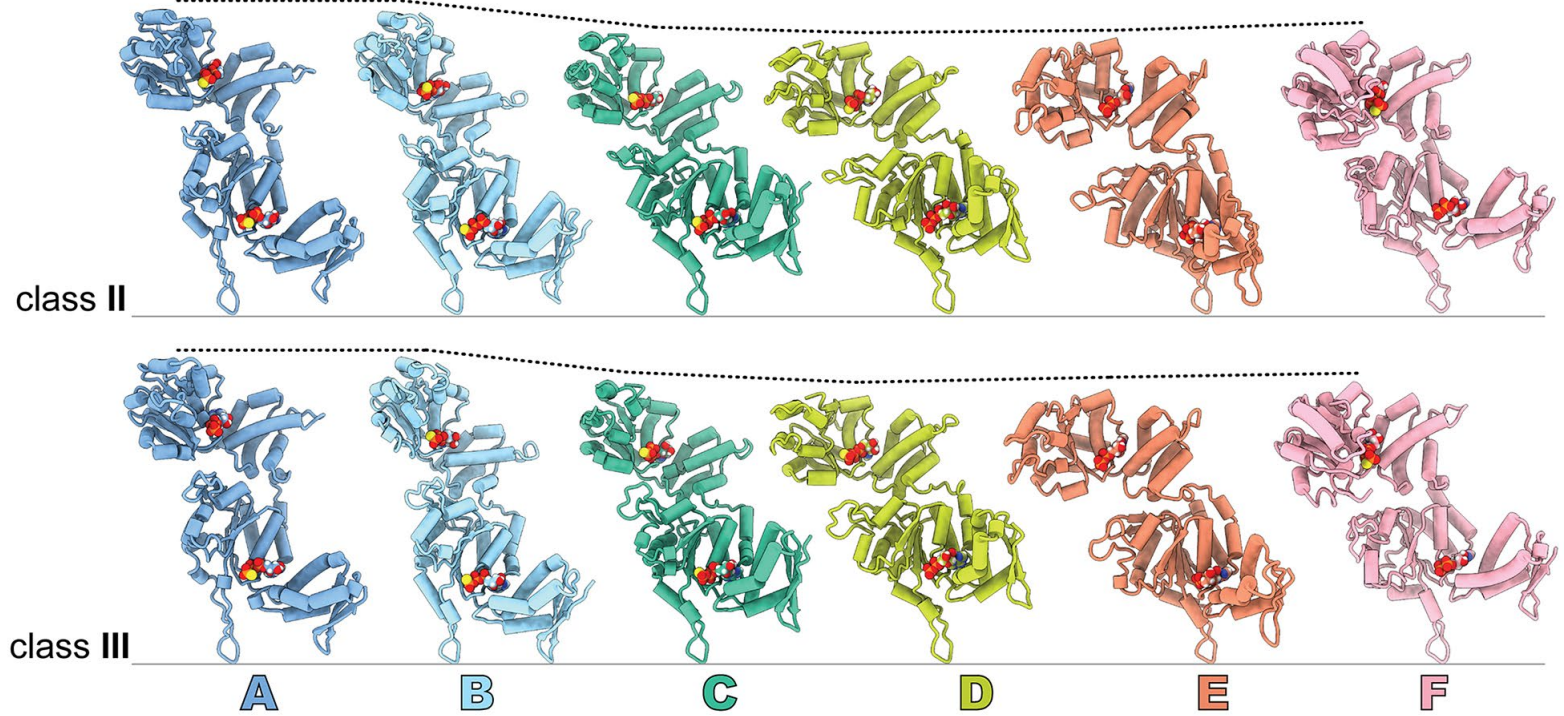

C

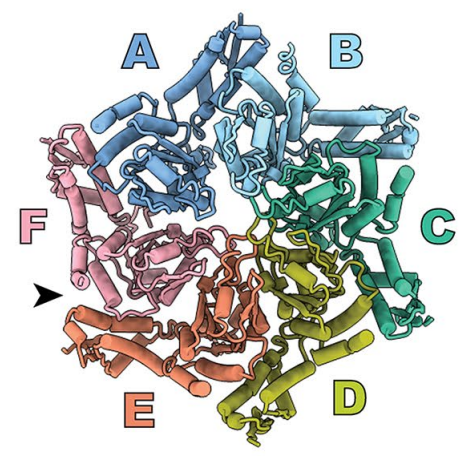

class-I D2

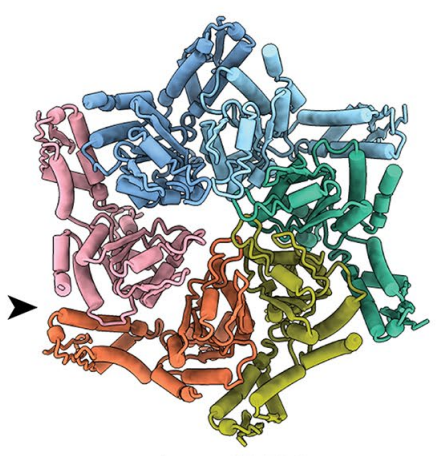

class-II D2

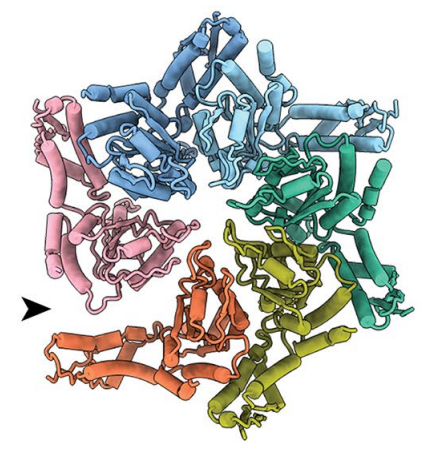

class-III D2

Figure 2. Conformational differences in ClpA subunits and hexamers

(A) Atomic models of class $\mathrm{I}, \mathrm{II}_{\mathrm{c}}$, and $\mathrm{III}_{\mathrm{a}} \mathrm{D} 1$ rings, showing position of subunits $\mathrm{A}$ and $\mathrm{B}$.

(B) Individual subunits of class $\mathbf{I}, \mathbf{I I}_{\mathbf{c}}$, and $\mathbf{I I I}_{\mathbf{a}}$ atomic models. The dashed line indicates the relative height of each subunit, following alignment to the bottom of the IGL loop.

(C) D2 ring rigid-body interface between subunits $\mathrm{E}$ and $\mathrm{F}$ of classes $\mathbf{I}, \mathbf{I I}_{\mathbf{c}}$, and $\mathrm{III}_{\mathrm{a}}$. In class $\mathrm{III}_{\mathrm{a}}$, the small $A A A+$ domain of subunit $E$ in the D2 ring swings out and loses contact (arrow) with the neighboring large $\mathrm{D} 2 \mathrm{AAA}+$ domain of subunit $\mathrm{F}$. 
A
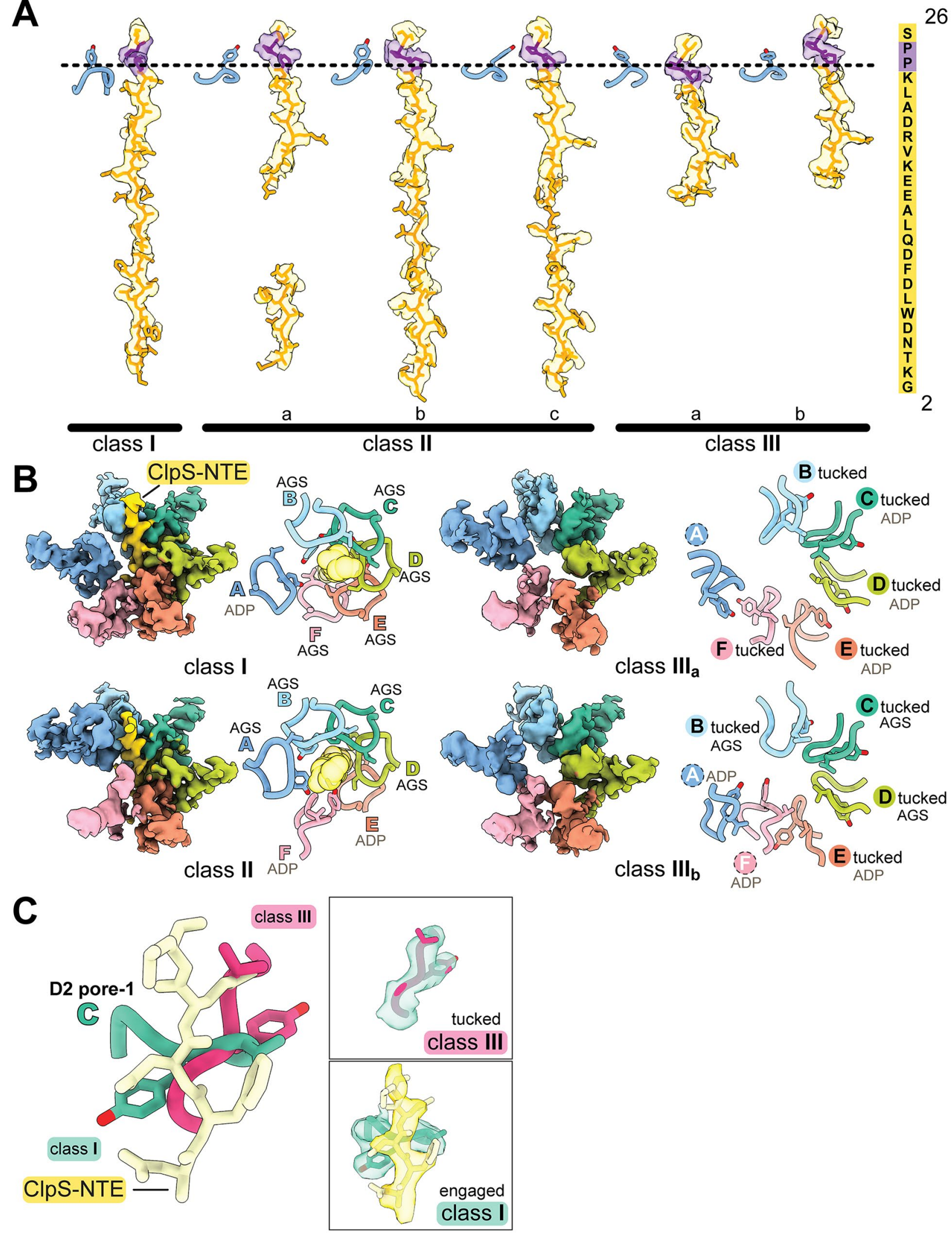


\section{Figure 3. Conformations of the CIpS NTE and D2 pore-1 loops of CIpA}

(A) Density of the ClpS NTE (transparent surface with modeled residues in sticks) in the ClpA axial channel in classes I, II, and III. Pro ${ }^{24}$ and Pro $^{25}$ (colored purple) are part of the junction sequence between the NTE and the ClpS core domain. The CIpS NTE sequence is shown on the right. The D1 pore-1 loop of subunit $A$ is shown as a reference point for the top of ClpA.

(B) D2 ring ClpA pore-1 loops and the CIpS NTE in classes $\mathbf{I}$, $\mathbf{I I}_{\mathbf{c}}$, and $\mathbf{I I I}_{\mathbf{a}, \mathbf{b}}$. The left panel in each structure depicts cryo-EM density for CIpA res. 528-555 and the ClpS NTE res. 2-15. The right panels are a zoomed-in view of the pore-1 loops (res. 538-542) and the NTE (transparent spheres), which is absent in class-III, in the atomic models. Subunit labels indicate nucleotide and interaction with the CIpS NTE. Labels in colored text denote NTE engagement; the dotted circle denotes lack of NTE engagement, with $\mathrm{Tyr}^{540}$ pointing towards the channel; labels in black text indicate the tucked conformation (Tyr ${ }^{540}$ away from the channel).

(C) Atomic models (sticks) and density (transparent surfaces in boxed area) of the subunit C D2 pore-1 loop in classes I (green) or III (pink) and the class-I CIpS NTE (yellow). In class III, density for the NTE is not observed. 


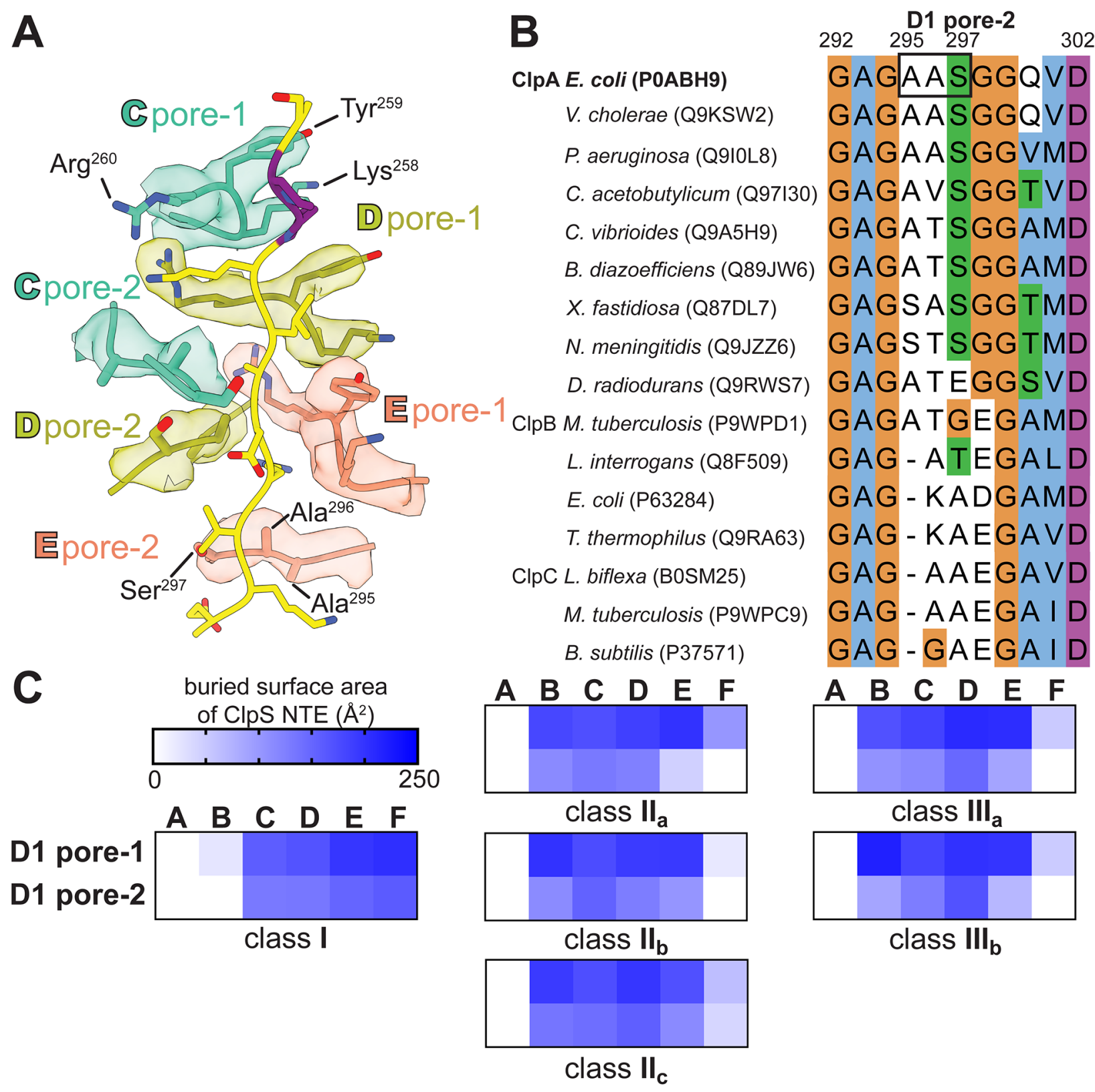

Figure 4. Interaction of CIpA pore-2 loops with the ClpS NTE in the D1 ring

(A) Three pairs of pore-1 (KYR, res. 258-260) and pore-2 (AAS, res. 295-297) loops in the ClpA D1 ring of class I, shown as sticks in representative subunit coloring, and the ClpS NTE (yellow and purple sticks). Cryo-EM density of each pore loop is shown with the respective transparent surfaces.

(B) Multiple sequence alignment of ClpABC family members corresponding to D1 pore-2 loops of E. coli ClpA (res. 292-302). UniProt accession numbers are listed in parentheses. The alignment at each position is colored according to ClustalX (orange=Gly, blue=hydrophobic, green=polar, magenta/purple=positive charge, white=unconserved).

(C) Buried surface area of the CIpS NTE by the pore-1 or pore-2 loops of the D1 ring in the atomic models of classes I, II, and III. See also Figure S7. 
A

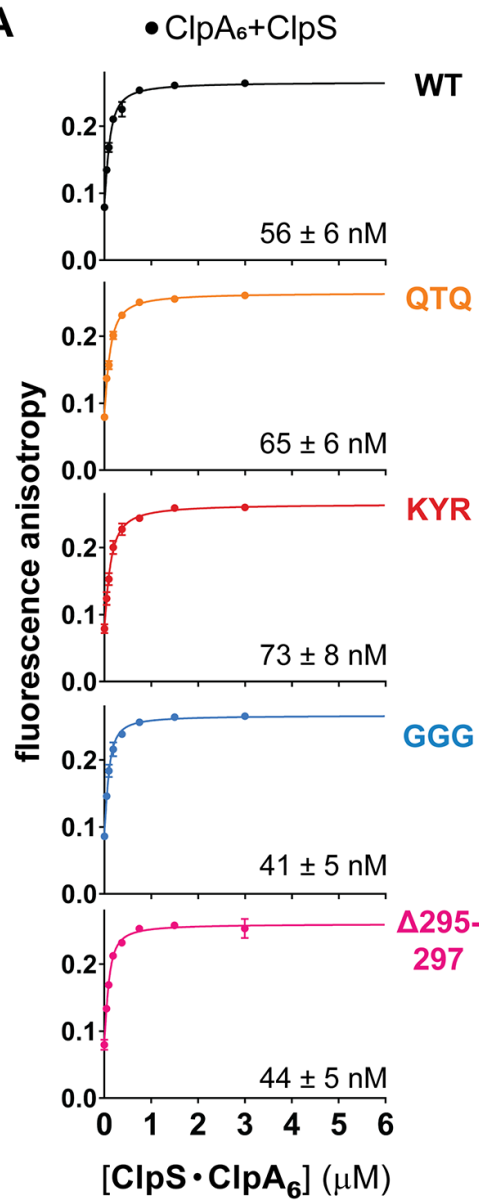

$\left[\mathrm{ClpS} \cdot \mathrm{ClpA}_{6}\right](\mu \mathrm{M})$ $\circ \mathrm{ClpA}_{6}$ only
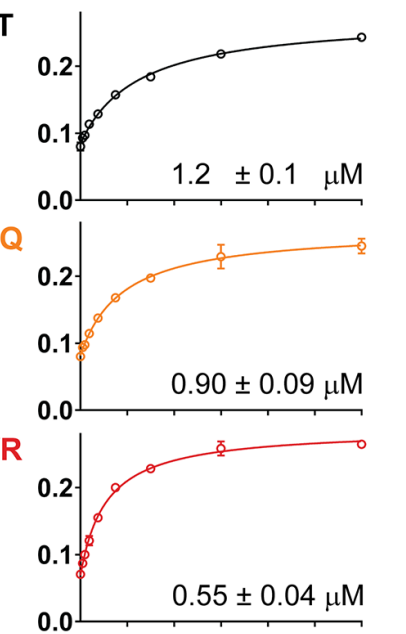

C

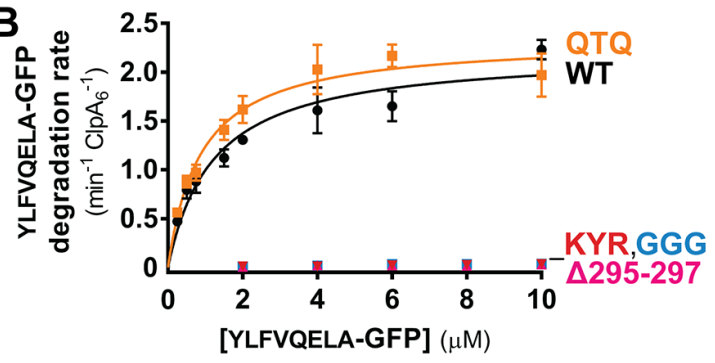

\begin{tabular}{|c|c|c|}
\hline pore-2 mutant & $\boldsymbol{K}_{\mathrm{M}}(\mu \mathrm{M})$ & $\mathbf{V}_{\max }\left(\min ^{-1} \mathrm{ClpA}_{6}^{-1}\right)$ \\
\hline WT (AAS) & $1.2 \pm 0.20$ & $2.2 \pm 0.11$ \\
\hline QTQ & $0.89 \pm 0.11$ & $2.3 \pm 0.09$ \\
\hline
\end{tabular}

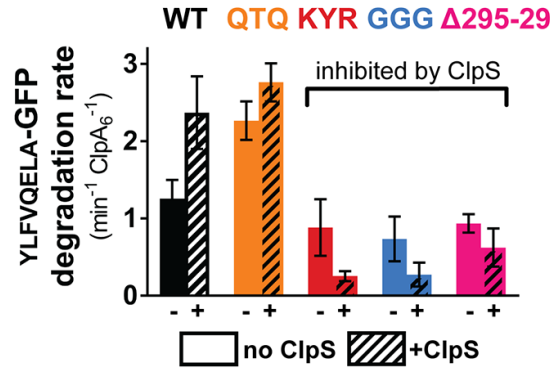

Figure 5. D1 pore-2 loops are critical for ClpS-mediated degradation

(A) Fluorescence anisotropy of ClpA pore-2 variants alone ( $\mathrm{ClpA}_{6}$, open circles), with an equimolar mixture with ClpS (+ClpS, filled circles), or ClpS only (bottom right panel), titrated in increasing concentrations against a fixed concentration of fluorescein-labeled $\mathrm{N}$-degron peptide (100 nM LLYVQRDSKEC-fl*) in the presence of $2 \mathrm{mM} \mathrm{ATP} \gamma \mathrm{S}$. Values are mean fluorescence anisotropy values from triplicates with error bars representing \pm 1 S.D, and were fit to equations listed in the Methods. The $\mathrm{K}_{d}$ values are reported on the lower right on each isotherm, with $( \pm)$ the standard error of nonlinear least-squares and $R^{2}$ values $=0.99$ for all fits.

(B) Kinetic analysis of YLFVQELA-GFP degradation by ClpA D1 pore-2 variants (see Methods for concentrations). Values are mean degradation rates $\left(\mathrm{min}^{-1} \mathrm{ClpA}_{6}{ }^{-1}\right)$ of triplicates with error bars representing $\pm 1 \mathrm{~S}$.D. In the table, $K_{\mathrm{M}}$ and $\mathrm{V}_{\max }$ values \pm errors were obtained by non-linear least squares fitting to the Michaelis-Menten equation. Degradation rates of GGG, $\Delta 295-297$, and KYR could not be fit to the Michaelis-Menten equation.

(C) YLFVQELA-GFP $(20 \mu \mathrm{M})$ degradation rates of ClpA D1 pore-2 variants $(0.1 \mu \mathrm{M} \mathrm{ClpA})$ and ClpP $\left(0.2 \mu \mathrm{M} \mathrm{ClpP}{ }_{14}\right)$, in the absence and presence of ClpS $(0.6 \mu \mathrm{M}$ ClpS $)$. Summary data are mean degradation rates $\left(\mathrm{min}^{-1} \mathrm{ClpA}_{6}{ }^{-1}\right)$ of triplicates with error bars representing \pm 1 S.D. 


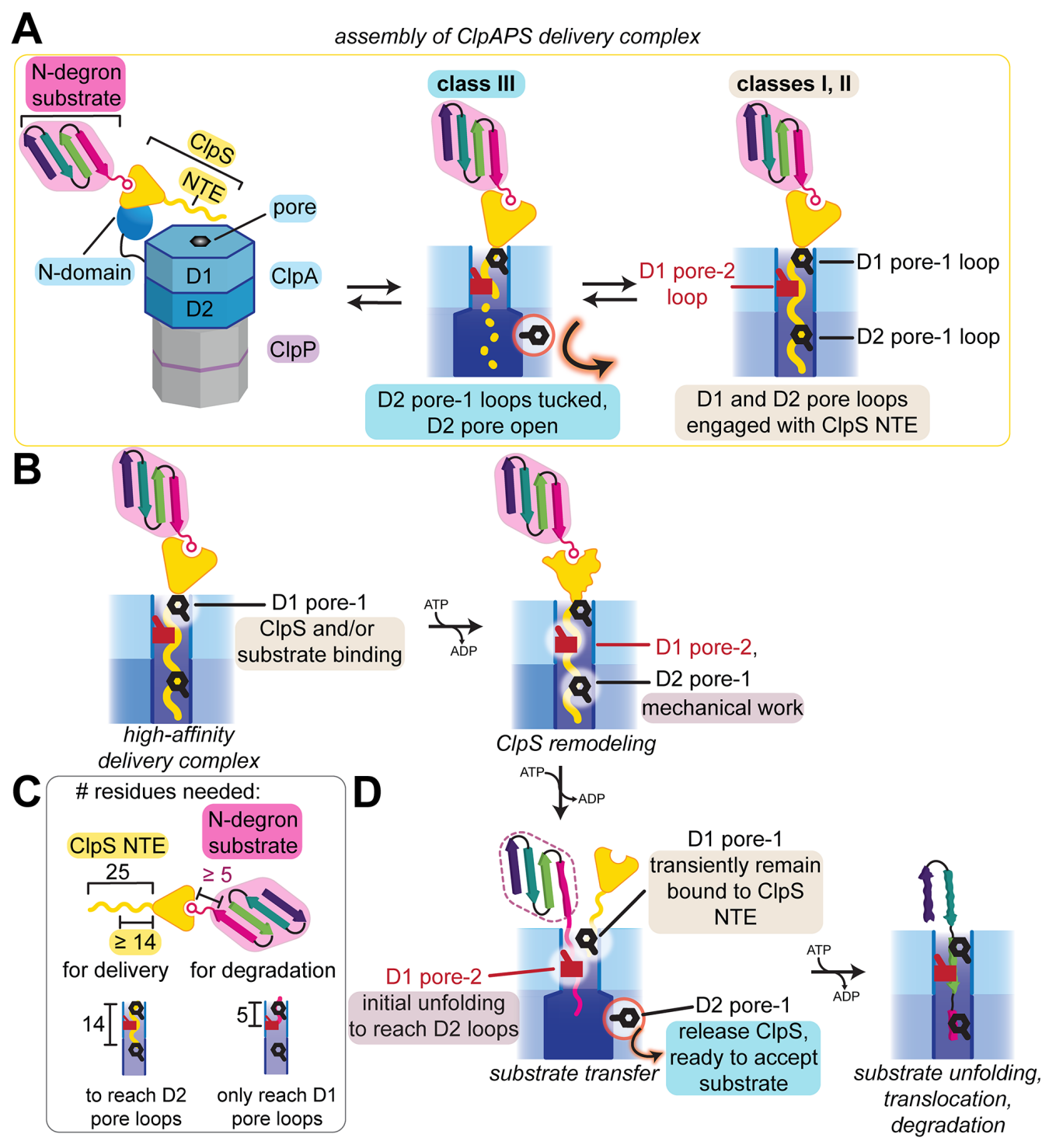

Figure 6. Models of CIpS-mediated degradation by CIpAP

(A) Summary of observed interactions of CIpS NTE and pore-1 and pore-2 loops in D1 and D2 from cryo-EM structures. In a dynamic equilibrium of low-affinity (not observed in this study) and high-affinity delivery complexes (classes I, II, and III), pore-1 loops in the D2 ring (i) are tucked-in and turned away from the axial channel (class III), correlated with a loss of observed CIpS NTE density, as indicated by the dotted line or (ii) contact the CIpS NTE (classes I, II).

(B) Pore loop functions in ClpS remodeling. Pore-1 loops, especially in D1, are required for CIpS and substrate binding to form delivery complexes. ATP hydrolysis powers ClpS remodeling, allowing D1 pore-2 and D2 pore-1 loops to translocate and tug on the ClpS NTE to promote substrate transfer and degradation.

(C) Summary from biochemical studies (Erbse et al., 2006; Wang et al., 2008b) of number of residues required for ClpS NTE and linker between N-end residue and folded domain in N-degron substrate.

(D) Proposed function of pore loops during substrate transfer. Following ClpS remodeling, D2 pore-1 loops may release the CIpS NTE, allowing for unfolding and translocation of the N-degron substrate to proceed from the D1 pore-2 loops. 


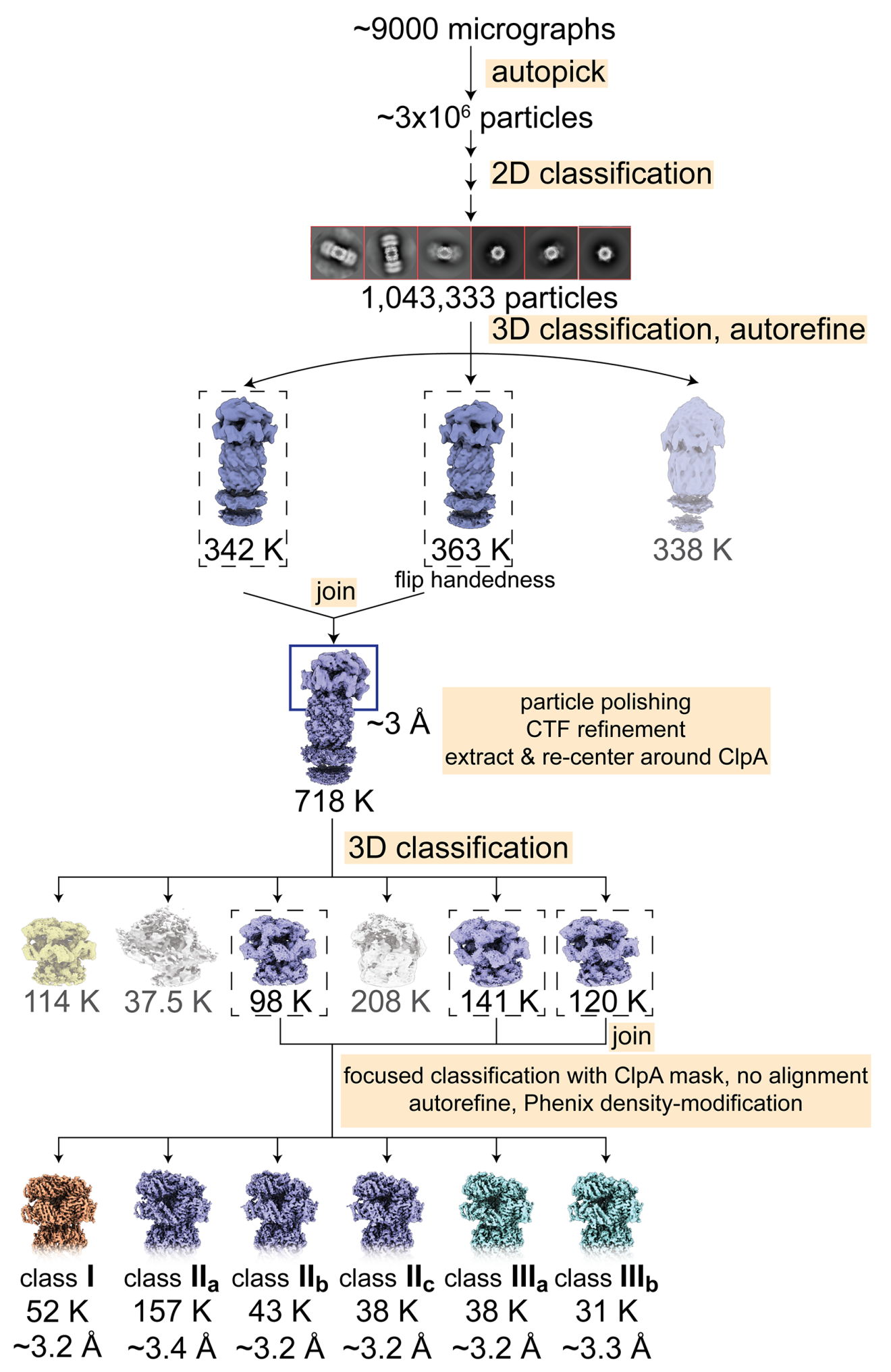

Figure S1. Cryo-EM data-processing workflow diagram, Related to Figure 1

EM micrographs containing doubly-capped ClpAP complexes (two ClpA hexamers bound to one ClpP 14-mer) were processed in RELION-3. The final 3D classes were refined using density-modification in PHENIX. 


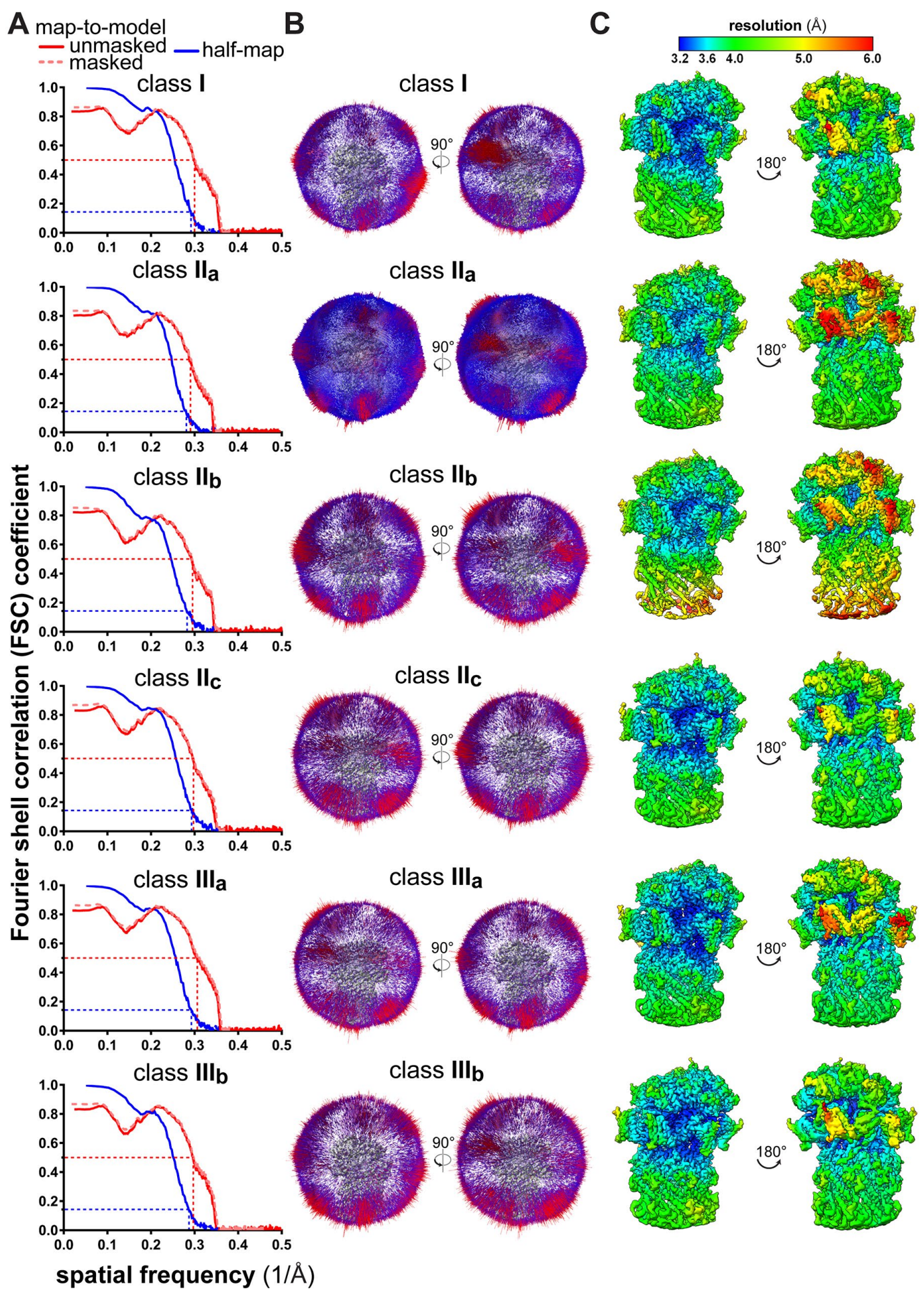

Figure S2. Cryo-EM validation, Related to Figure 1

(A) Fourier Shell Correlation (FSC) plots of half maps (shown in blue) or model-map (shown in red) resolution. The dashed lines indicate the cut-off values at $\mathrm{FSC}=0.5$ (model-map) or $\mathrm{FSC}=0.143$ (half-map).

(B) Euler angle distribution plots of the particles used in the final reconstruction of class $\mathbf{I}, \mathbf{I I}_{\mathrm{a}-\mathrm{c}}$, and $\mathrm{III}_{\mathrm{a}, \mathrm{b}}$ structures.

(C) Local resolution maps of final reconstructions, colored according to RELION-3 calculations. 


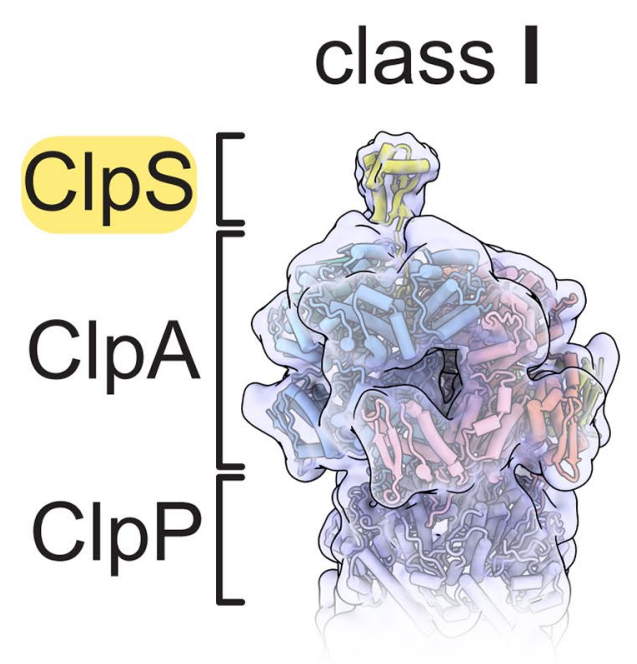

\section{class II}

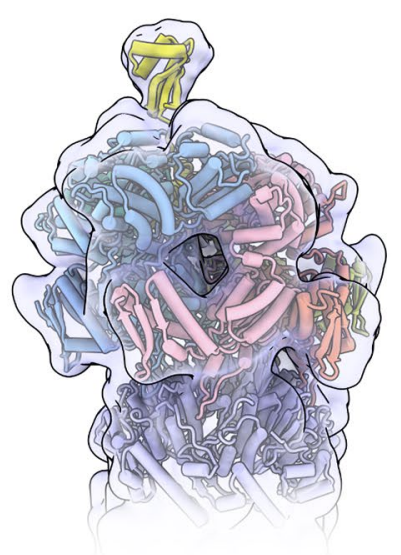

a

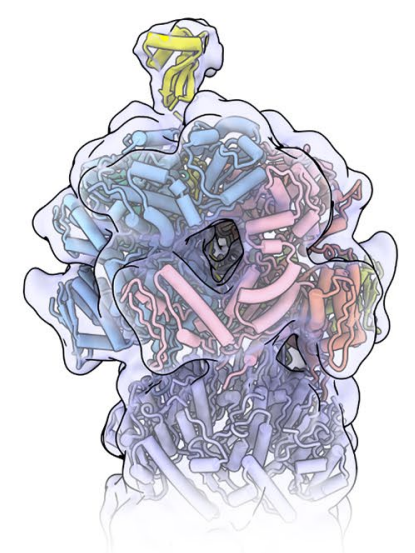

b

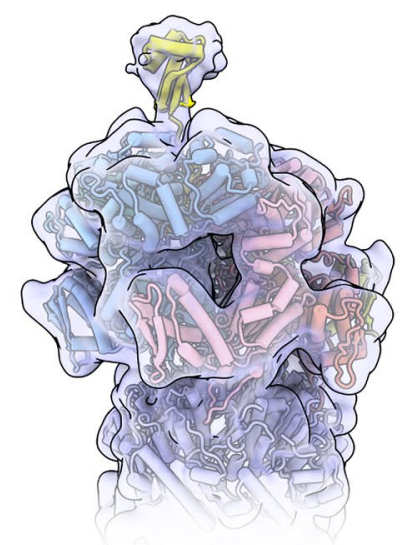

a

b

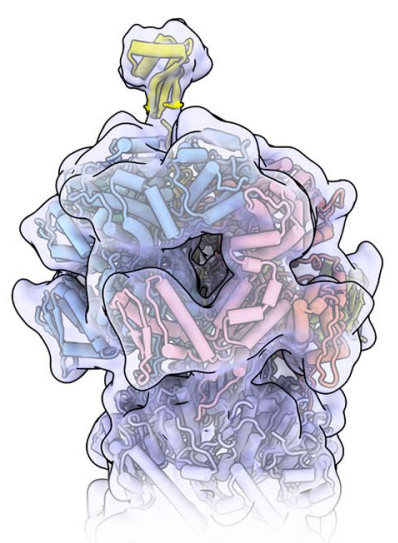

C

Figure S3. ClpS docking to cryo-EM maps, Related to Figure 1

The ClpS core domain (PDB 3O2B; res. 27-106) was docked to final reconstructions of class I, $\mathrm{II}_{\mathrm{a}-\mathrm{c}}$, and $\mathrm{III}_{\mathrm{a}, \mathrm{b}}$ that were low-pass filtered to $10 \AA$. 


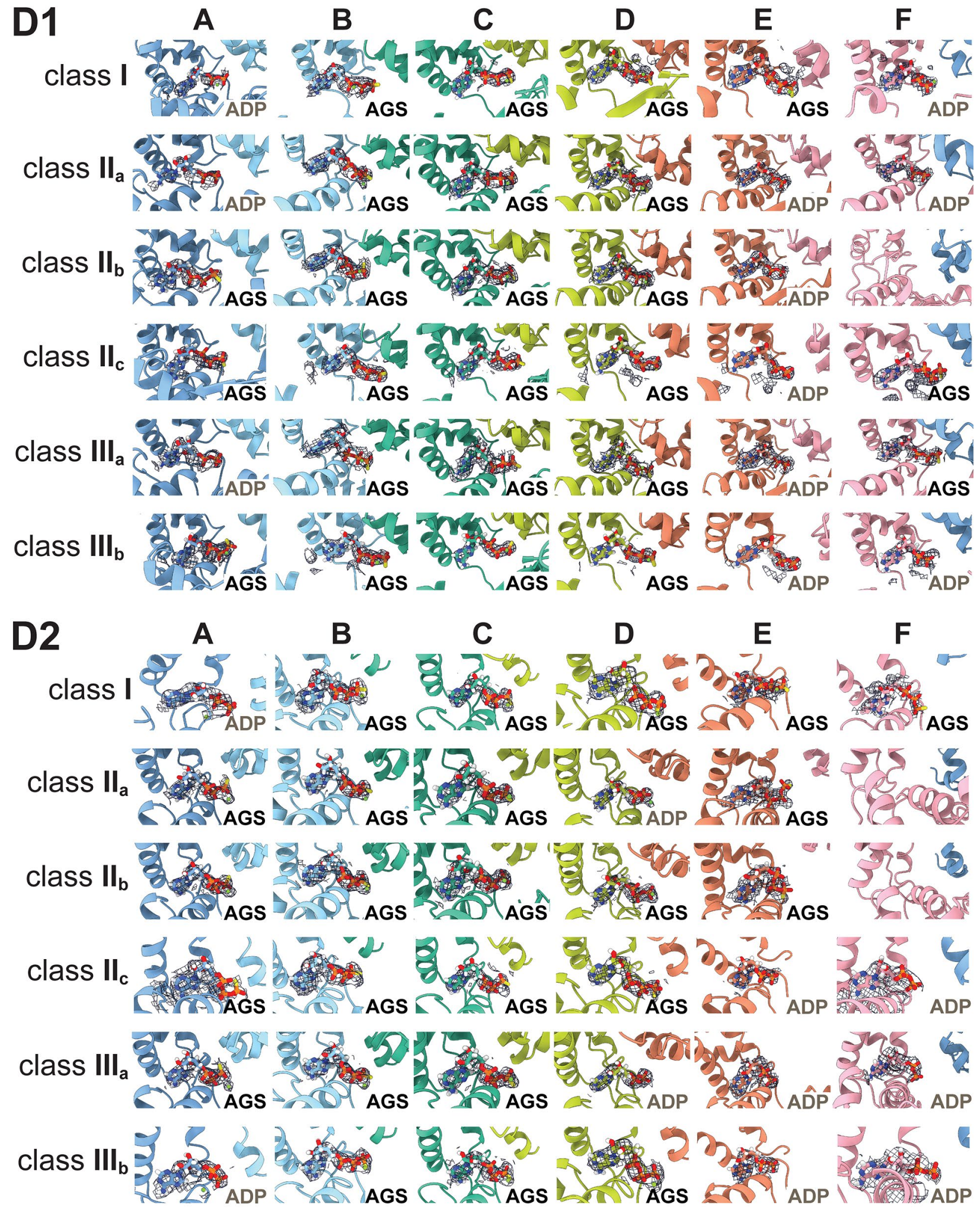

Figure S4. Nucleotide occupancy, Related to Figure 2, Related to Figure 3

EM density (shown as mesh) of nucleotides in each D1 and D2 binding site of class $\mathbf{I}$, $\mathbf{I I}_{\mathrm{a}-\mathrm{c}}$, and $\mathrm{III}_{\mathbf{a}, \mathrm{b}}$ structures. Nucleotide density is not observed in the D1 site of $\mathrm{F}$ subunit in $\mathrm{II}_{\mathrm{b}}$ or the D2 site of the $\mathrm{F}$ subunit of $\mathrm{II}_{\mathbf{a}}$ or $\mathrm{II}_{\mathbf{b}}$. 


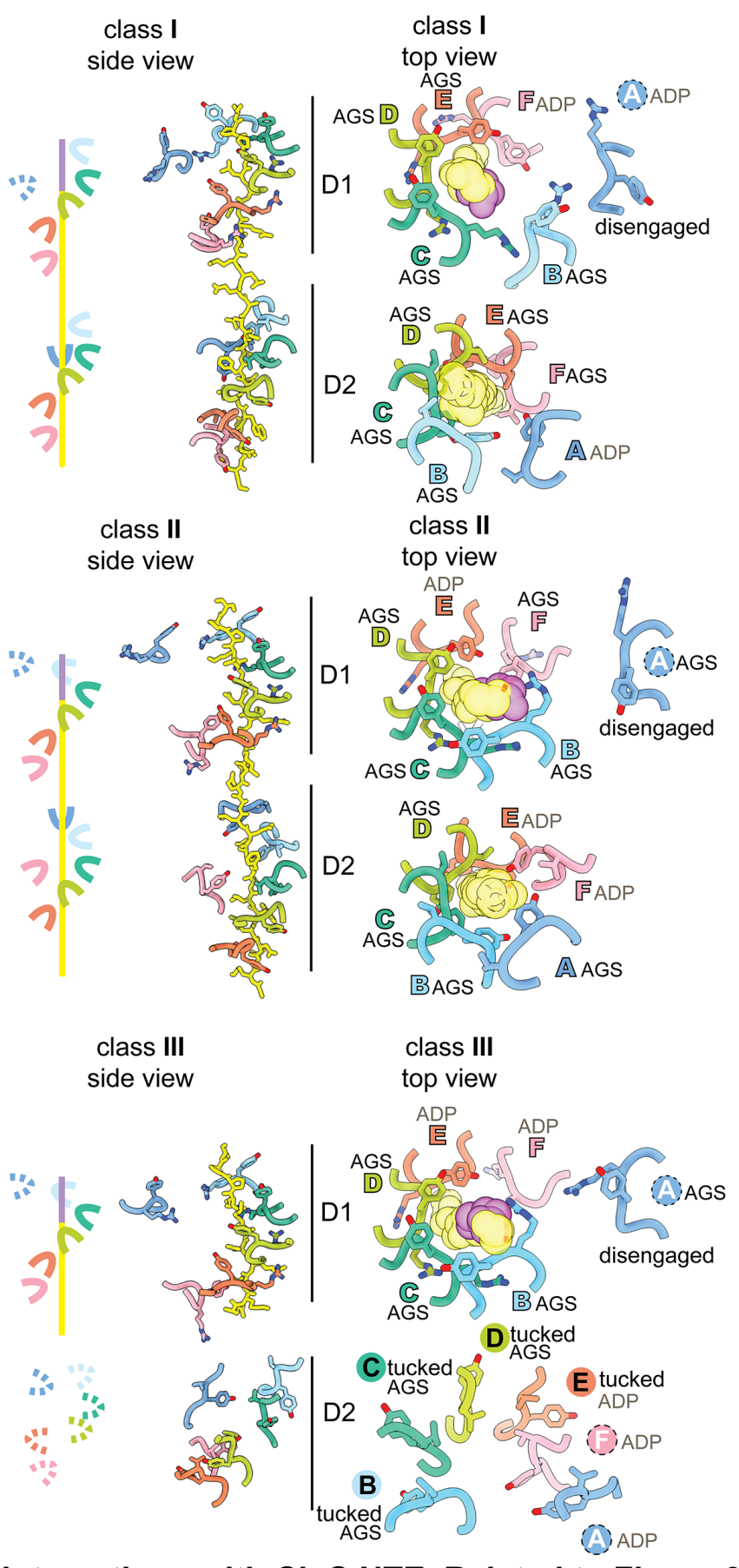

Figure S5. Pore-1 loop interactions with CIpS NTE, Related to Figure 3B-C

The leftmost panel in each structure is a diagram of pore-1 loops in the D1 and D2 rings, where solid lines represent the presence of NTE contacts and dashed lines represent their absence. Middle panel shows lateral views of contacts between ClpA pore-1 loops and the CIpS NTE in class $\mathbf{I}, \mathbf{I I}_{\mathbf{c}}$, and $\mathbf{I I}_{\mathbf{b}}$ atomic models. The rightmost panel in each structure is a closer view of the pore-1 loops with the NTE shown as transparent spheres and the corresponding nucleotide from each subunit. Labels in colored text denote NTE engagement; the dotted circle denotes lack of NTE engagement, with $\mathrm{Tyr}^{540}$ pointing towards the channel; labels in black text indicate the tucked conformation ( $\mathrm{Ty} \mathrm{r}^{540}$ away from the channel). 


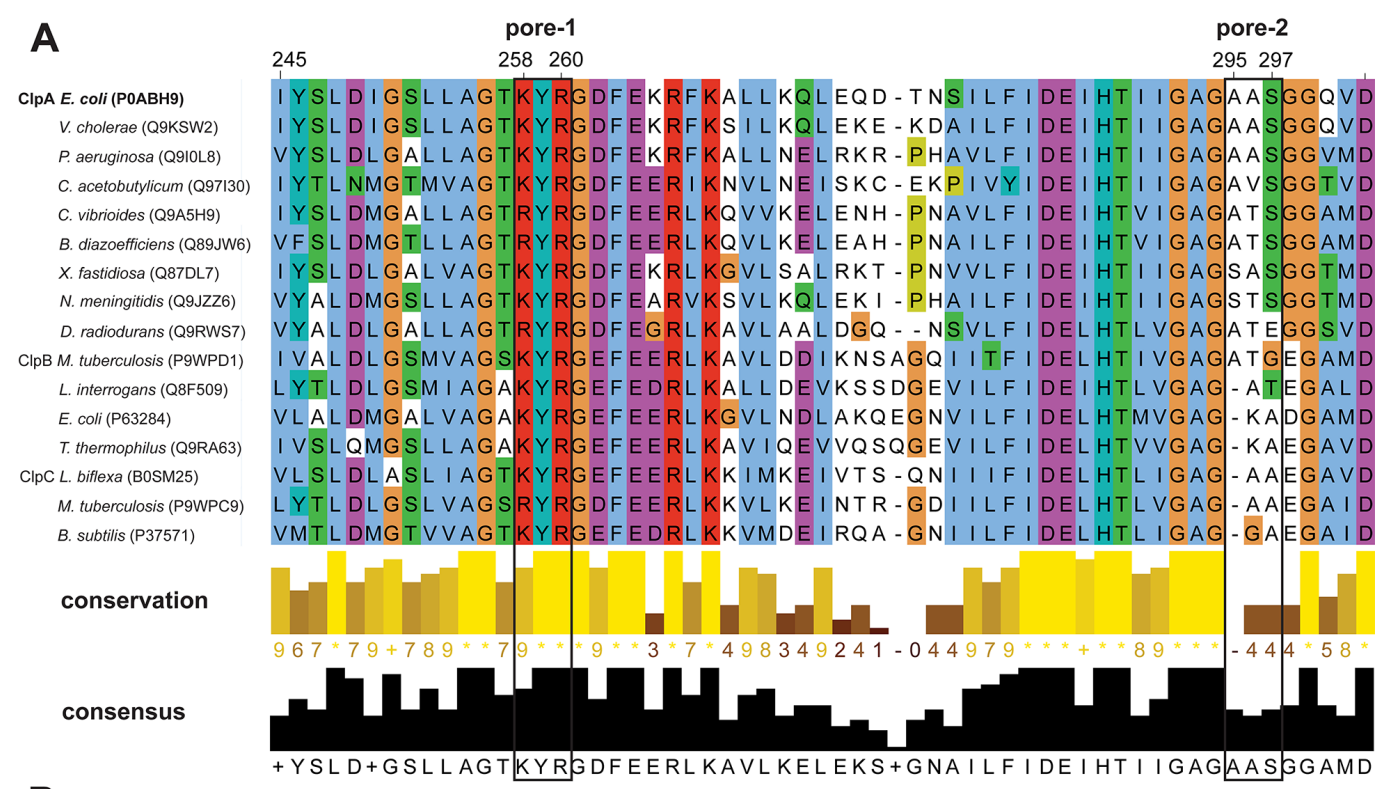

B

class I
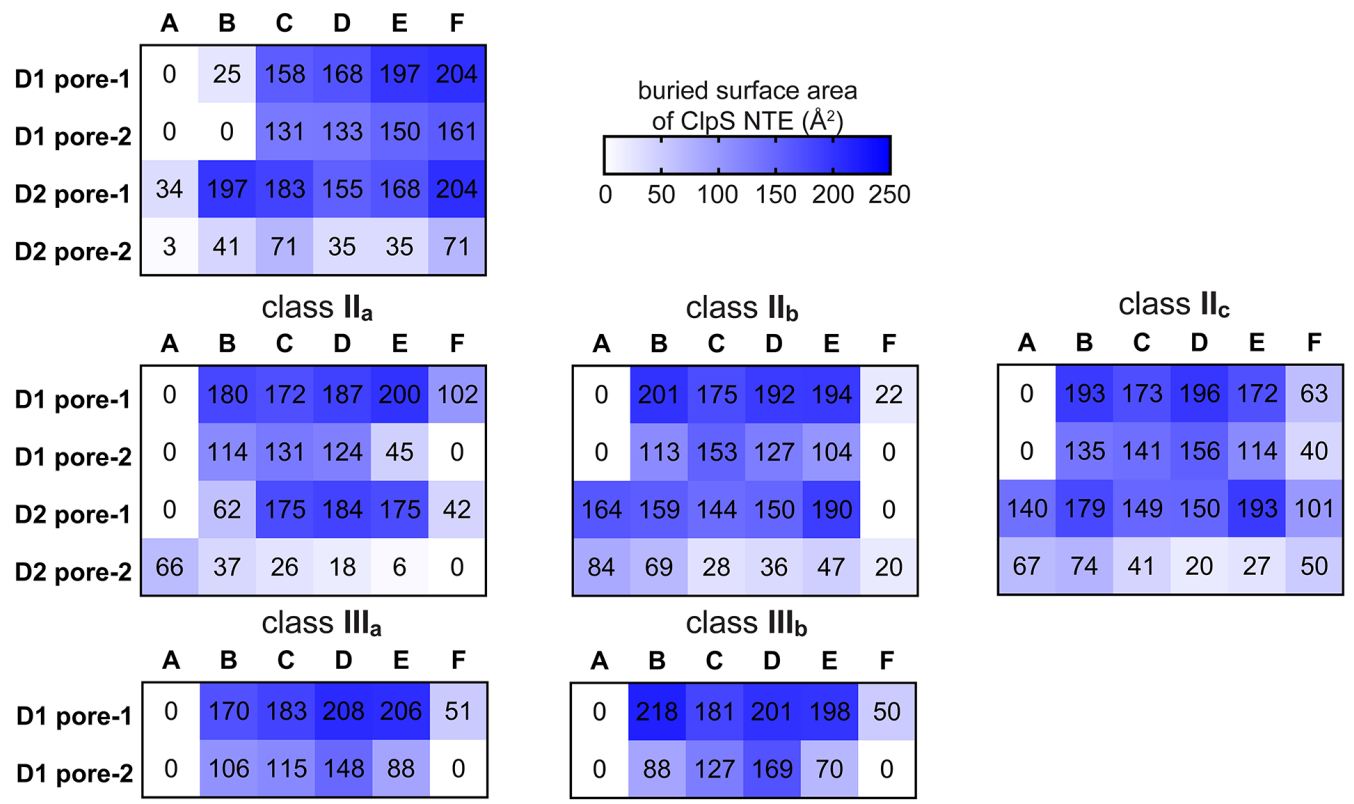

Figure S6. Comparison of pore-1 and pore-2 loop sequences and contacts to CIpS NTE, Related to Figure 4B-C

(A) Multiple sequence alignment of ClpABC family members, corresponding to pore-1 (res. 258-260, E. coli ClpA) and D1 pore-2 loops of E. coli ClpA (res. 292-302), created using MUSCLE alignment (Edgar, 2004). UniProt accession numbers are listed in parentheses. The alignment at each position is colored according to ClustalX (orange=Gly, blue=hydrophobic, green=polar, magenta/purple=positive charge, white=unconserved). Conservation scores are calculated in Jalview from the amino acid properties in the alignment. Conserved columns that have the highest conservation score are indicated by '*' symbols (corresponding to a numeric score of 11), followed next by mutations that conserve all physico-chemical properties, indicated by '+' symbols. Gaps are indicated by '-', and the lowest conservation score is zero.

(B) Buried Surface Area (BSA) of ClpS NTE. Contacts between CIpS NTE and pore-1 or pore-2 loops in the D1 and D2 rings of classes $\mathbf{I}, \mathbf{I l}_{\mathbf{a}-\mathrm{c}}$, and $\mathbf{I I}_{\mathbf{a}, \mathbf{b}}$ were evaluated using PISA. Raw BSA values are provided in each box that correspond to coloring by the heat map scale. 

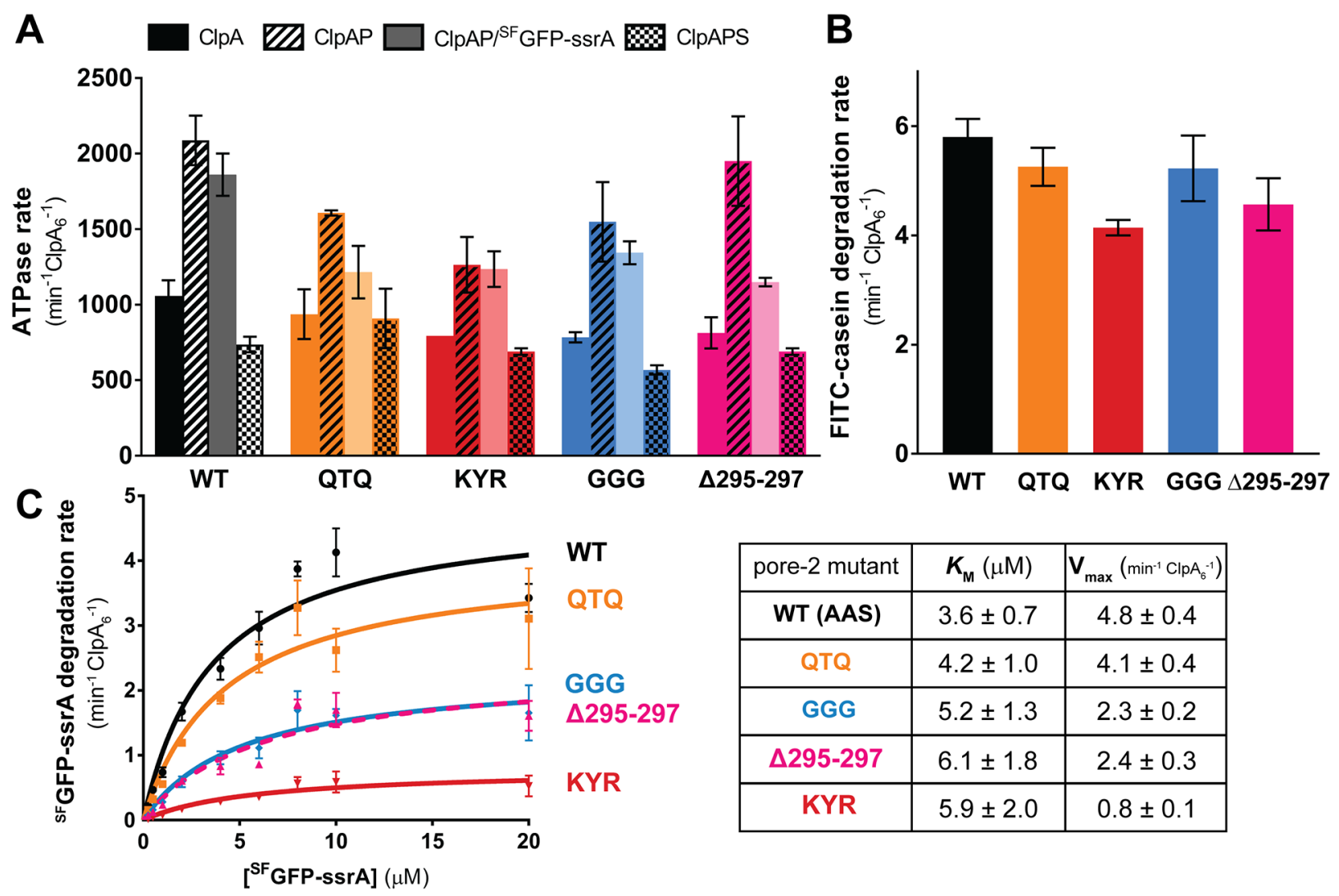

\begin{tabular}{|c|c|c|}
\hline pore-2 mutant & $\boldsymbol{K}_{\mathrm{M}}(\mu \mathrm{M})$ & $\mathbf{V}_{\max }\left(\min ^{-1} \mathrm{ClPA}_{6}{ }^{-1}\right)$ \\
\hline WT (AAS) & $3.6 \pm 0.7$ & $4.8 \pm 0.4$ \\
\hline QTQ & $4.2 \pm 1.0$ & $4.1 \pm 0.4$ \\
\hline GGG & $5.2 \pm 1.3$ & $2.3 \pm 0.2$ \\
\hline$\Delta 295-297$ & $6.1 \pm 1.8$ & $2.4 \pm 0.3$ \\
\hline KYR & $5.9 \pm 2.0$ & $0.8 \pm 0.1$ \\
\hline
\end{tabular}

Figure S7. D1 pore-2 loop variants can support ATP hydrolysis and substrate unfolding, translocation, and degradation, Related to Figure 5

(A) ATP hydrolysis rates of ClpA D1 pore-2 variants alone or in the presence of ClpP, ClpP and SFGFP-ssrA substrate, or ClpP and ClpS; see Methods for concentrations.

(B) FITC-casein $(20 \mu \mathrm{M})$ degradation rates of ClpA D1 pore-2 variants $(0.2 \mu \mathrm{M} \mathrm{ClpA}$ ) and ClpP $\left(0.4 \mu \mathrm{M} \mathrm{ClpP}_{14}\right)$. Summary data are mean degradation rates $\left(\mathrm{min}^{-1} \mathrm{ClpA}_{6}{ }^{-1}\right)$ of triplicates with error bars representing \pm 1 S.D.

(C) Kinetic analysis of ${ }^{S F}$ GFP-ssrA degradation by ClpA D1 pore-2 variants $(0.2 \mu \mathrm{M}$ ClpA, $0.4 \mu \mathrm{M}$ $\left.\mathrm{ClpP}_{14}\right)$. Values are mean degradation rates $\left(\mathrm{min}^{-1} \mathrm{ClpA}_{6}{ }^{-1}\right)$ of triplicates with error bars representing \pm 1 S.D. In the table, $K_{\mathrm{M}}$ and $V_{\max }$ values \pm errors were obtained by non-linear least squares fitting to the Michaelis-Menten equation. 


\section{METHODS}

\section{Proteins and peptides}

ClpA pore-2 mutations were introduced using round-the-horn mutagenesis with T4 polynucleotide kinase and Q5 high-fidelity polymerase (New England Biolabs) into pET9a-M169TCIpA, the plasmid used to express ClpA (gift from J. Flanagan, Hershey Medical Center). The M169T substitution helps overexpression of full-length CIpA (Seol et al., 1995) and is present in our lab version of 'wild-type' CIpA. CIpA and pore-2 variants were purified as described (Hou et al., 2008) and stored in $\mathrm{HO}$ buffer (50 mM HEPES-KOH [pH 7.5], $300 \mathrm{mM} \mathrm{NaCl,} 20 \mathrm{mM} \mathrm{MgCl}$, $10 \%$ [w/v] glycerol, 0.5 mM DTT). ClpP-His 6 was expressed in E. coli strain JK10 (clpP::cat, $\Delta / o n$, slyD::kan, ADE3 (Kenniston et al., 2003)), purified as described (Kim et al., 2000), and stored in $50 \mathrm{mM} \mathrm{Tris-HCl}$ (pH 8), $150 \mathrm{mM} \mathrm{KCl,} \mathrm{10 \%} \mathrm{glycerol,} 0.5$ mM EDTA, and $1 \mathrm{mM}$ DTT. CIpS and YLFVQELA-GFP were expressed in E. coli strain BL21(DE3) and purified as described for $\mathrm{HiS}_{6}$-SUMO-fusion proteins (Hou et al., 2008; Román-Hernández et al., 2011). SFGFP-ssrA was expressed and purified as described (Nager et al., 2011). ClpS, SFGFP-ssrA, and YLFVQELA-GFP were stored in 25 mM HEPES-KOH (pH 7.5), 150 mM KCl, 10\% glycerol, and 1 mM DTT. FITC-casein (Sigma-Aldrich C0528) was dissolved in $\mathrm{HO}$ buffer and used freshly for biochemical assays; an extinction coefficient at $280 \mathrm{~nm}\left(11,460 \mathrm{M}^{-1} \mathrm{~cm}^{-1}\right)$ and absorbance values at $280 \mathrm{~nm}$ and $494 \mathrm{~nm}$ (to calculate and correct for overlap from the fluorescence of the FITC moiety) were used to calculate its concentration. The LLYVQRDSKEC-fluorescein N-degron synthetic peptide (21st Century Biochemicals [Marlborough, MA], molecular weight $1779.9 \mathrm{~g} / \mathrm{mol}$ ) was dissolved and stored at $100 \mu \mathrm{M}$ in $15 \%$ DMSO.

\section{Sample preparation and EM data acquisition}

$\mathrm{ClpA}_{6}(4 \mu \mathrm{M}), \mathrm{ClpP}_{14}(8 \mu \mathrm{M}), \mathrm{ClpS}(13 \mu \mathrm{M})$, and YLFVQELA-GFP $(13 \mu \mathrm{M})$ were mixed in $70 \mu \mathrm{L}$ of assembly buffer (50 mM HEPES-KOH, pH 7.5, $300 \mathrm{mM} \mathrm{KCl,} 5 \mathrm{mM} \mathrm{MgCl}$, 2 mM TCEP, 4\% glycerol, $2 \mathrm{mM} \mathrm{ATP} \gamma \mathrm{S}$ [Calbiochem]) for $5 \mathrm{~min}$ at $25{ }^{\circ} \mathrm{C} .25 \mu \mathrm{L}$ of this mixture was then 
chromatographed at room temperature and a flow rate of $0.04 \mathrm{~mL} / \mathrm{min}$ on a Superdex-200 3.2/300 size exclusion column equilibrated in assembly buffer (GE Healthcare Ettan). A $50 \mu \mathrm{L}$ fraction containing the largest molecular weight complex was assessed by SDS-PAGE (stained with SYPRO Red [Thermo Fisher]) and pooled for cryo-EM. After diluting the sample two-fold in assembly buffer, a $3 \mu \mathrm{L}$ aliquot of the mixture was applied to glow-discharged R1.2/1.3 300 mesh holey carbon gold grids (Quantifoil). After a $15 \mathrm{~s}$ incubation, grids were blotted for $4 \mathrm{~s}$ at $4{ }^{\circ} \mathrm{C}, 100$ $\%$ humidity, using Whatman grade 595 filter paper, and plunged into liquid ethane using a Vitrobot Mark IV system (Thermo Fisher Scientific).

A single grid was imaged for data collection using a Talos Arctica with a Gatan K3 direct electron detector (University of Massachusetts Chan Medical School Cryo-EM Microscopy Facility, Worcester, MA) in super-resolution mode, operated at $200 \mathrm{keV}$ to collect high-resolution movies at $(0.435 \AA$ per pixel; uncalibrated magnification $45,000 X)$ with a defocus range of $-0.5 \mu \mathrm{m}$ to -2.5 $\mu \mathrm{m}$, with a total dose of $34.71 \mathrm{e}-/ \AA^{2}$ over 26 frames (200 ms per frame).

\section{Cryo-EM data processing}

Each movie was binned by a factor of 2 , aligned, corrected for beam-induced motion using MotionCor2 (Zheng et al., 2017), and CTF estimation was calculated by CTFFIND4 (Rohou and Grigorieff, 2015). A total of 9,169 micrographs were analyzed using RELION 3.0.8 (Zivanov et al., 2018) for data processing, classification, and 3D reconstruction. The majority of auto-picked particles were doubly capped complexes consisting of two CIpA hexamers per ClpP 14-mer. Following three rounds of 2D classification, 1,043,033 particles were used for 3D reconstruction. The cryo-EM map of CIpXP (EMD-20406), which was collected under similar parameters as our dataset, was low-pass filtered to $60 \AA$ to generate an initial model for reconstruction. After the first round of $3 \mathrm{D}$ classification, two of three high-quality classes were combined, totaling 717,833 particles; the second class closely resembled the first with the exception of handedness and was flipped to correct handedness before being combined. The two classes were also utilized for per- 
particle CTF refinement and motion correction. The combined class had a resolution of $\sim 3 \AA$. The fulcrum was shifted to the center of ClpA, and particles were re-boxed to improve the resolution of this region of CIpA before performing the second round of 3D classification $(T=4)$ with alignment to generate six classes. Three good classes were selected and combined (358,726 particles) for a third round of 3D classification with a ClpA mask, without alignment $(T=20)$ to yield the final six classes. Each class was then subjected to 3D auto-refinement without symmetry to yield six maps with $\sim 3.5 \AA$ resolution. To generate the final maps, each map was density-modified and autosharpened in PHENIX (Adams et al., 2010), giving final resolutions of ranging from 3.2-3.4

\section{A (Table 1).}

\section{Molecular modeling and refinement}

The CIpAP cryo-EM structure (PDB code 6W23) was docked into the EM map for the class-I structure, and the CIpAP cryo-EM structure (PDB code 6W22) was docked into all other EM maps using "fit in map" in Chimera (Pettersen et al., 2004). Real-space refinement was performed using PHENIX, and model building was performed in Coot (Emsley et al., 2004). The ClpS NTE sequence (residues 2-26) was added manually in Coot. Geometry of the final models was evaluated using MolProbity (Williams et al., 2018). Figures and movies were generated using Chimera, ChimeraX (Goddard et al., 2007), and PyMOL (Schrödinger, LLC).

\section{Multiple sequence alignment}

The amino-acid sequences of bacterial CIpA, CIpB, and ClpC proteins were downloaded from UniProtKB (Bateman et al., 2021) and aligned using MUSCLE (Edgar, 2004) with MEGA7 (Kumar et al., 2016). The sequence alignment was visualized in Jalview (Waterhouse et al., 2009) and colored according to the Clustal X scheme.

\section{Buried surface area calculations}


The buried surface area of the ClpS NTE in all class structures was analyzed using the 'Protein interfaces, surfaces and assemblies' service PISA at the European Bioinformatics Institute. (http://www.ebi.ac.uk/pdbe/prot_int/pistart.html). The BSA values were summed from the D1 pore-1 loop region (residues 254-264), D1 pore-2 loop region (residues 292-302), D2 pore-1 loop region (residues 536-544), and D2 pore-2 loop region (residues 525-531).

\section{Biochemical assays}

Biochemical experiments were performed with at least three technical replicates at $30{ }^{\circ} \mathrm{C}$ in $\mathrm{HO}$ buffer using a SpectraMax M5 Microplate Reader (Molecular Devices) to measure initial rates of absorbance or fluorescence changes or equilibrium anisotropy values. ATP-hydrolysis rates were measured over the first $\sim 2$ min by monitoring the loss of absorbance at $340 \mathrm{~nm}$ using a coupled NADH-ATP assay (Burton et al., 2001) with 5 mM ATP (Sigma-Aldrich), pyruvate kinase (SigmaAldrich; P9136 at 20 units $/ \mathrm{mL}$ ), lactate dehydrogenase (Sigma-Aldrich; L1254 at 20 units/mL), 7.5 mM phosphoenolpyruvate (Sigma-Aldrich; P0564), and 0.2 mM NADH (Roche 10107735001). ATP-hydrolysis assays were performed under four conditions: (i) $\mathrm{ClpA}_{6}$ or variants $(0.2 \mu \mathrm{M})$; (ii) ClpA $_{6}$ or variants $(0.1 \mu \mathrm{M})$ and $\mathrm{ClpP}_{14}(0.1 \mu \mathrm{M})$; (iii) $\mathrm{ClpA}_{6}$ or variants $(0.2 \mu \mathrm{M}), \mathrm{ClpP}_{14}(0.2 \mu \mathrm{M})$,

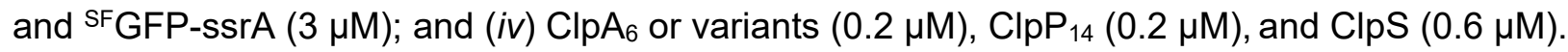

FITC-casein degradation assays were monitored by increases in fluorescence (excitation $340 \mathrm{~nm}$, emission $520 \mathrm{~nm}$ ) as a consequence of protease-dependent unquenching over the first $5 \mathrm{~min}$; reactions contained $\mathrm{ClpA}_{6}$ or variants $(0.2 \mu \mathrm{M}), \mathrm{ClpP}_{14}(0.4 \mu \mathrm{M})$, and an ATP-regeneration system (4 mM ATP, $50 \mu \mathrm{g} / \mathrm{mL}$ creatine kinase [Sigma-Aldrich], $5 \mathrm{mM}$ creatine phosphate [Sigma-Aldrich]). The endpoint fluorescence for complete FITC-casein degradation was determined by addition of porcine elastase $(100 \mu \mathrm{g} / \mathrm{mL}$; Sigma-Aldrich) to each well, followed by a 30 -min incubation prior to reading. To determine FITC-casein degradation rates, the increase in relative fluorescence units was normalized to the endpoint fluorescence value from fully unquenched substrate after 
porcine elastase incubation and the background rate was subtracted from each reaction on the basis of a no-enzyme buffer-only control.

Degradation of GFP variants was monitored by loss of fluorescence (excitation $467 \mathrm{~nm}$, emission $511 \mathrm{~nm}$ ) over the first 5-10 min. Briefly, rates were calculated by normalizing the slope values of relative fluorescence units (RFUs)/time by the fluorescence signal determined from a standard curve of RFUs versus varying concentrations of substrate in the linear range. Degradation of different concentrations of SFGFP-ssrA $(0.25-20 \mu \mathrm{M})$ was assayed using $\mathrm{ClpA}_{6}$ or variants $(0.2$ $\mu \mathrm{M}), \mathrm{ClpP}_{14}(0.4 \mu \mathrm{M})$, and the ATP-regeneration system described above. Degradation of different concentrations of YLFVQELA-GFP $(0.25-20 \mu \mathrm{M})$ was assayed using $\mathrm{ClpA}_{6}$ or variants $(0.1 \mu \mathrm{M})$, $\mathrm{ClpP}_{14}(0.2 \mu \mathrm{M})$, ClpS $(0.6 \mu \mathrm{M})$, and the ATP-regeneration system. For degradation of YLFVQELA-GFP by the GGG, KYR, and $\Delta 295-297$ pore-2-loop mutants shown in Figure 5B, concentrations were $\mathrm{ClpA}_{6}$ variant $(0.6 \mu \mathrm{M}), \mathrm{ClpP}_{14}(1.2 \mu \mathrm{M})$, and ClpS $(3.6 \mu \mathrm{M})$.

The binding of the peptide LLYVQRDSKEC-fluorescein $(100 \mathrm{nM})$ to $(i) \mathrm{ClpA}_{6}$ or variants $(0.047-6$ $\mu \mathrm{M})$, (ii) ClpS (1.25 - $30 \mu \mathrm{M})$, or (iii) equimolar mixtures of $\mathrm{ClpA}_{6}$ or variants and ClpS $(0.047-3$ $\mu \mathrm{M})$ at equilibrium was assayed by fluorescence anisotropy (excitation $490 \mathrm{~nm}$, emission $525 \mathrm{~nm}$ ) in the presence of $\operatorname{ATP} \gamma \mathrm{S}(2 \mathrm{mM})$. Only $\mathrm{ClpA}_{6} \cdot \mathrm{ClpS} \cdot$ peptide ternary and $\mathrm{ClpA}_{6} \cdot$ peptide binary complexes have higher anisotropy levels (in comparison to ClpS•peptide binary complexes) as a result of the much larger molecular weight of $\mathrm{ClpA}_{6}(\sim 500 \mathrm{kDa})$ compared to that of ClpS $(\sim 10$ kDa). Data were fit by a non-linear least-squares algorithm to equations for $\mathrm{ClpA}_{6}$ only and $\mathrm{ClpS}$ only experiments:

$$
\text { fluorescence anisotropy }=f_{\min }+\left(\frac{f_{\max } * K_{d}}{K_{d}+X}\right)
$$

or to a quadratic equation for tight binding for $\mathrm{ClpA}_{6} \cdot \mathrm{ClpS}$ complexes:

$$
\text { fluorescence anisotropy }=f_{\text {min }}+\left(f_{\max }-f_{\text {min }}\right) \frac{\left(L+X+K_{d}\right)-\sqrt{\left(L+X+K_{d}\right)^{2}-4 \cdot L \cdot X}}{2 L}
$$


bioRxiv preprint doi: https://doi.org/10.1101/2022.02.04.479121; this version posted February 4, 2022. The copyright holder for this preprint (which was not certified by peer review) is the author/funder. All rights reserved. No reuse allowed without permission.

where $f_{\min }$ is the background anisotropy value, $f_{\max }$ is the maximum anisotropy value at saturated binding, $L$ is the concentration of peptide $(100 \mathrm{nM}), K_{\mathrm{d}}$ is the dissociation equilibrium constant (in $\mathrm{nM}$ ), and $X$ is the concentration of $\mathrm{ClpA}_{6} \cdot \mathrm{ClpS}$ (in nM). 


\section{REFERENCES}

Adams, P.D., Afonine, P. V, Bunkóczi, G., Chen, V.B., Davis, I.W., Echols, N., Headd, J.J., Hung, L.-W., Kapral, G.J., Grosse-Kunstleve, R.W., et al. (2010). PHENIX: a comprehensive Pythonbased system for macromolecular structure solution. Acta Crystallogr. D. Biol. Crystallogr. 66, Pt 2, 213-221.

Alfieri, C., Chang, L., and Barford, D. (2018). Mechanism for remodelling of the cell cycle checkpoint protein MAD2 by the ATPase TRIP13. Nature 559, 7713, 274-278.

Barkow, S.R., Levchenko, I., Baker, T.A., and Sauer, R.T. (2009). Polypeptide translocation by the AAA+ CIpXP protease machine. Chem. Biol. 16, 6, 605.

Bateman, A., Martin, M.-J., Orchard, S., Magrane, M., Agivetova, R., Ahmad, S., Alpi, E., BowlerBarnett, E.H., Britto, R., Bursteinas, B., et al. (2021). UniProt: the universal protein knowledgebase in 2021. Nucleic Acids Res. 49, D1, D480-D489.

Bell, T.A., Baker, T.A., and Sauer, R.T. (2019). Interactions between a subset of substrate side chains and AAA+ motor pore loops determine grip during protein unfolding. eLife 8, e46808.

Blok, N.B., Tan, D., Wang, R.Y.-R., Penczek, P.A., Baker, D., DiMaio, F., Rapoport, T.A., and Walz, T. (2015). Unique double-ring structure of the peroxisomal Pex1/Pex6 ATPase complex revealed by cryo-electron microscopy. Proc. Natl. Acad. Sci. U. S. A. 112, 30, E4017-25.

Bodnar, N., and Rapoport, T. (2017a). Toward an understanding of the Cdc48/p97 ATPase. F1000Research 6, 1318.

Bodnar, N.O., and Rapoport, T.A. (2017b). Molecular mechanism of substrate processing by the Cdc48 ATPase complex. Cell 169, 4, 722-735.e9.

Burton, R.E., Siddiqui, S.M., Kim, Y.I., Baker, T.A., and Sauer, R.T. (2001). Effects of protein stability and structure on substrate processing by the CIpXP unfolding and degradation machine. EMBO J. 20, 12, 3092-3100.

Cooney, I., Han, H., Stewart, M.G., Carson, R.H., Hansen, D.T., Iwasa, J.H., Price, J.C., Hill, C.P., and Shen, P.S. (2019). Structure of the Cdc48 segregase in the act of unfolding an authentic substrate. Science. 365, 6452, 502-505.

Daskalogianni, C., Apcher, S., Candeias, M.M., Naski, N., Calvo, F., and Fåhraeus, R. (2008). Gly-Ala repeats induce position- and substrate-specific regulation of $26 \mathrm{~S}$ proteasome-dependent partial processing. J. Biol. Chem. 283, 44, 30090-30100.

Deville, C., Carroni, M., Franke, K.B., Topf, M., Bukau, B., Mogk, A., and Saibil, H.R. (2017). Structural pathway of regulated substrate transfer and threading through an Hsp100 disaggregase. Sci. Adv. 3, 8, e1701726.

De Donatis, G.M., Singh, S.K., Viswanathan, S., and Maurizi, M.R. (2010). A single ClpS monomer is sufficient to direct the activity of the ClpA hexamer. J. Biol. Chem. 285, 12, 87718781.

Dong, Y., Zhang, S., Wu, Z., Li, X., Wang, W.L., Zhu, Y., Stoilova-McPhie, S., Lu, Y., Finley, D., and Mao, Y. (2019). Cryo-EM structures and dynamics of substrate-engaged human 26S proteasome. Nature 565, 7737, 49-55.

Dougan, D.A., Reid, B.G., Horwich, A.L., and Bukau, B. (2002). ClpS, a substrate modulator of the ClpAP machine. Mol. Cell 9, 3, 673-683. 
Doyle, S.M., Hoskins, J.R., and Wickner, S. (2012). DnaK chaperone-dependent disaggregation by caseinolytic peptidase $\mathrm{B}(\mathrm{ClpB})$ mutants reveals functional overlap in the $\mathrm{N}$-terminal domain and nucleotide-binding domain-1 pore tyrosine. J. Biol. Chem. 287, 34, 28470-28479.

Edgar, R.C. (2004). MUSCLE: multiple sequence alignment with high accuracy and high throughput. Nucleic Acids Res. 32, 5, 1792-1797.

Effantin, G., Ishikawa, T., De Donatis, G.M., Maurizi, M.R., and Steven, A.C. (2010). Local and global mobility in the CIpA AAA+ chaperone detected by cryo-electron microscopy: Functional connotations. Structure 18, 5, 553-562.

Emsley, P., Cowtan, K., and IUCr (2004). Coot: model-building tools for molecular graphics. Acta Crystallogr. Sect. D Biol. Crystallogr. 60, 12, 2126-2132.

Erbse, A., Schmidt, R., Bornemann, T., Schneider-Mergener, J., Mogk, A., Zahn, R., Dougan, D.A., and Bukau, B. (2006). ClpS is an essential component of the $\mathrm{N}$-end rule pathway in Escherichia coli. Nature 439, 7077, 753-756.

Erzberger, J.P., and Berger, J.M. (2006). Evolutionary relationships and structural mechanisms of AAA+ proteins. Annu. Rev. Biophys. Biomol. Struct. 35, 93-114.

Fei, X., Bell, T.A., Jenni, S., Stinson, B.M., Baker, T.A., Harrison, S.C., and Sauer, R.T. (2020a). Structures of the ATP-fueled CIpXP proteolytic machine bound to protein substrate. eLife 9, e52774.

Fei, X., Bell, T.A., Barkow, S.R., Baker, T.A., and Sauer, R.T. (2020b). Structural basis of ClpXP recognition and unfolding of ssra-tagged substrates. eLife 9, e61496.

Gardner, B.M., Castanzo, D.T., Chowdhury, S., Stjepanovic, G., Stefely, M.S., Hurley, J.H., Lander, G.C., and Martin, A. (2018). The peroxisomal AAA-ATPase Pex1/Pex6 unfolds substrates by processive threading. Nat. Commun. 9, 1, 135.

Gates, S.N., Yokom, A.L., Lin, J., Jackrel, M.E., Rizo, A.N., Kendsersky, N.M., Buell, C.E., Sweeny, E.A., Mack, K.L., Chuang, E., et al. (2017). Ratchet-like polypeptide translocation mechanism of the AAA+ disaggregase Hsp104. Science. 357, 6348, 273-279.

Goddard, T.D., Huang, C.C., and Ferrin, T.E. (2007). Visualizing density maps with UCSF Chimera. J. Struct. Biol. 157, 1, 281-287.

Grimaud, R., Kessel, M., Beuron, F., Steven, A.C., and Maurizi, M.R. (1998). Enzymatic and structural similarities between the Escherichia coli ATP- dependent proteases, CIpXP and CIpAP. J. Biol. Chem. 273, 20, 12476-12481.

Guo, F., Esser, L., Singh, S.K., Maurizi, M.R., and Xia, D. (2002). Crystal structure of the heterodimeric complex of the adaptor, ClpS, with the N-domain of the AAA+ chaperone, ClpA. J. Biol. Chem. 277, 48, 46753-46762.

Han, H., Fulcher, J.M., Dandey, V.P., Iwasa, J.H., Sundquist, W.I., Kay, M.S., Shen, P.S., and Hill, C.P. (2019). Structure of Vps4 with circular peptides and implications for translocation of two polypeptide chains by AAA+ ATPases. eLife 8, e44071.

Han, H., Schubert, H.L., McCullough, J., Monroe, N., Purdy, M.D., Yeager, M., Sundquist, W.I., and Hill, C.P. (2020). Structure of spastin bound to a glutamate-rich peptide implies a hand-overhand mechanism of substrate translocation. J. Biol. Chem. 295, 2, 435-443.

Hattendorf, D.A., and Lindquist, S.L. (2002). Cooperative kinetics of both Hsp104 ATPase 
domains and interdomain communication revealed by AAA sensor-1 mutants. EMBO J. 21, 1-2, 12-21.

Hinnerwisch, J., Fenton, W.A., Furtak, K.J., Farr, G.W., and Horwich, A.L. (2005a). Loops in the central channel of ClpA chaperone mediate protein binding, unfolding, and translocation. Cell 121, 7, 1029-1041.

Hinnerwisch, J., Reid, B.G., Fenton, W.A., and Horwich, A.L. (2005b). Roles of the N-domains of the ClpA unfoldase in binding substrate proteins and in stable complex formation with the ClpP protease. J. Biol. Chem. 280, 49, 40838-40844.

Hoskins, J.R., Pak, M., Maurizi, M.R., and Wickner, S. (1998). The role of the ClpA chaperone in proteolysis by CIpAP. Proc. Natl. Acad. Sci. U. S. A. 95, 21, 12135-12140.

Hoskins, J.R., Singh, S.K., Maurizi, M.R., and Wickner, S. (2000). Protein binding and unfolding by the chaperone ClpA and degradation by the protease ClpAP. Proc. Natl. Acad. Sci. U. S. A. 97, 16, 8892-8897.

Hou, J.Y., Sauer, R.T., and Baker, T.A. (2008). Distinct structural elements of the adaptor ClpS are required for regulating degradation by ClpAP. Nat. Struct. Mol. Biol. 15, 3, 288-294.

Hoyt, M.A., Zich, J., Takeuchi, J., Zhang, M., Govaerts, C., and Coffino, P. (2006). Glycine-alanine repeats impair proper substrate unfolding by the proteasome. EMBO J. 25, 8, 1720-1729.

Iosefson, O., Olivares, A.O., Baker, T.A., and Sauer, R.T. (2015). Dissection of axial-pore loop function during unfolding and translocation by a AA+ proteolytic machine. Cell Rep. 12, 6, 10321041.

Ishikawa, T., Beuron, F., Kessel, M., Wickner, S., Maurizi, M.R., and Steven, A.C. (2001). Translocation pathway of protein substrates in ClpAP protease. Proc. Natl. Acad. Sci. U. S. A. 98, $8,4328-4333$.

Johjima, A., Noi, K., Nishikori, S., Ogi, H., Esaki, M., and Ogura, T. (2015). Microtubule severing by katanin p60 AAA+ATPase requires the $C$-terminal acidic tails of both $\alpha$-and $\beta$-tubulins and basic amino acid residues in the AAA+ring pore. J. Biol. Chem. 290, 18, 11762-11770.

Kavalchuk, M., Jomaa, A., Müller, A.U., and Weber-Ban, E. (2022). Structural basis of prokaryotic ubiquitin-like protein engagement and translocation by the mycobacterial Mpa-proteasome complex. Nat. Commun. 2022131 13, 1, 1-13.

Kenniston, J.A., Baker, T.A., Fernandez, J.M., and Sauer, R.T. (2003). Linkage between ATP consumption and mechanical unfolding during the protein processing reactions of an AAA+ degradation machine. Cell 114, 4, 511-520.

Kim, Y.I., Burton, R.E., Burton, B.M., Sauer, R.T., and Baker, T.A. (2000). Dynamics of substrate denaturation and translocation by the ClpXP degradation machine. Mol. Cell 5, 4, 639-648.

Kotamarthi, H.C., Sauer, R.T., and Baker, T.A. (2020). The non-dominant AAA+ ring in the CIpAP protease functions as an anti-stalling motor to accelerate protein unfolding and translocation. Cell Rep. 30, 8, 2644-2654.e3.

Kraut, D.A. (2013). Slippery substrates impair ATP-dependent protease function by slowing unfolding. J. Biol. Chem. 288, 48, 34729-34735.

Kraut, D.A., Israeli, E., Schrader, E.K., Patil, A., Nakai, K., Nanavati, D., Inobe, T., and Matouschek, A. (2012). Sequence- and species-dependence of proteasomal processivity. ACS 
Chem. Biol. 7, 8, 1444-1453.

Kress, W., Mutschler, H., and Weber-Ban, E. (2009). Both ATPase domains of ClpA are critical for processing of stable protein structures. J. Biol. Chem. 284, 45, 31441-31452.

Krissinel, E., and Henrick, K. (2007). Inference of macromolecular assemblies from crystalline state. J. Mol. Biol. 372, 3, 774-797.

Kumar, S., Stecher, G., and Tamura, K. (2016). MEGA7: Molecular evolutionary genetics analysis version 7.0 for bigger datasets. Mol. Biol. Evol. 33, 7, 1870-1874.

Levitskaya, J., Sharipo, A., Leonchiks, A., Ciechanover, A., and Masucci, M.G. (1997). Inhibition of ubiquitin/proteasome-dependent protein degradation by the Gly-Ala repeat domain of the Epstein-Barr virus nuclear antigen 1. Proc. Natl. Acad. Sci. U. S. A. 94, 23, 12616-12621.

Lin, L., and Ghosh, S. (1996). A glycine-rich region in NF-kappaB p105 functions as a processing signal for the generation of the p50 subunit. Mol. Cell. Biol. 16, 5, 2248-2254.

Lo, Y.-H.H., Sobhany, M., Hsu, A.L., Ford, B.L., Krahn, J.M., Borgnia, M.J., and Stanley, R.E. (2019). Cryo-EM structure of the essential ribosome assembly AAA-ATPase Rix7. Nat. Commun. $10,1,513$.

Lopez, K.E., Rizo, A.N., Tse, E., Lin, J., Scull, N.W., Thwin, A.C., Lucius, A.L., Shorter, J., and Southworth, D.R. (2020). Conformational plasticity of the ClpAP AAA+ protease couples protein unfolding and proteolysis. Nat. Struct. Mol. Biol. 27, 5, 406-416.

Mahmoud, S.A., and Chien, P. (2018). Regulated proteolysis in bacteria. Annu. Rev. Biochem. 87, 677-696

Martin, A., Baker, T.A., and Sauer, R.T. (2008). Pore loops of the AAA+ CIpX machine grip substrates to drive translocation and unfolding. Nat. Struct. Mol. Biol. 15, 11, 1147-1151.

Miller, J.M., and Lucius, A.L. (2014). ATPYS competes with ATP for binding at Domain 1 but not Domain 2 during ClpA catalyzed polypeptide translocation. Biophys. Chem. 185, 58-69.

Mogk, A., Schlieker, C., Strub, C., Rist, W., Weibezahn, J., and Bukau, B. (2003). Roles of individual domains and conserved motifs of the AAA+ chaperone ClpB in oligomerization, ATP hydrolysis, and chaperone activity. J. Biol. Chem. 278, 20, 17615-17624.

Nager, A.R., Baker, T.A., and Sauer, R.T. (2011). Stepwise unfolding of a $\beta$ barrel protein by the AAA+ ClpXP protease. J. Mol. Biol. 413, 1, 4-16.

Olivares, A.O., Baker, T.A., and Sauer, R.T. (2015). Mechanistic insights into bacterial AAA+ proteases and protein-remodelling machines. Nat. Rev. Microbiol. 14, 1, 33-44.

Pettersen, E.F., Goddard, T.D., Huang, C.C., Couch, G.S., Greenblatt, D.M., Meng, E.C., and Ferrin, T.E. (2004). UCSF Chimera--a visualization system for exploratory research and analysis. J. Comput. Chem. 25, 13, 1605-1612.

Puchades, C., Rampello, A.J., Shin, M., Giuliano, C.J., Wiseman, R.L., Glynn, S.E., and Lander, G.C. (2017). Structure of the mitochondrial inner membrane AAA+ protease YME1 gives insight into substrate processing. Science. 358, 6363.

Puchades, C., Ding, B., Song, A., Wiseman, R.L., Lander, G.C., and Glynn, S.E. (2019). Unique structural features of the mitochondrial AAA+ protease AFG3L2 reveal the molecular basis for activity in health and disease. Mol. Cell 75, 5, 1073-1085.e6. 
Puchades, C., Sandate, C.R., and Lander, G.C. (2020). The molecular principles governing the activity and functional diversity of AAA+ proteins. Nat. Rev. Mol. Cell Biol. 21, 1, 43-58.

Ripstein, Z.A., Huang, R., Augustyniak, R., Kay, L.E., and Rubinstein, J.L. (2017). Structure of a $A A A+$ unfoldase in the process of unfolding substrate. eLife 6, e25754.

Ripstein, Z.A., Vahidi, S., Houry, W.A., Rubinstein, J.L., and Kay, L.E. (2020). A processive rotary mechanism couples substrate unfolding and proteolysis in the CIpXP degradation machinery. eLife 9, e52158.

Rivera-Rivera, I. (2015). Mechanism of active substrate delivery by the AAA+ protease adaptor ClpS. Ph.D. thesis, Massachusetts Institute of Technology. Retrieved from https://dspace.mit.edu/handle/1721.1/101352

Rivera-Rivera, I., Román-Hernández, G., Sauer, R.T., and Baker, T.A. (2014). Remodeling of a delivery complex allows ClpS-mediated degradation of N-degron substrates. Proc. Natl. Acad. Sci. U. S. A. 111, 37, E3853-9.

Rizo, A.N., Lin, J.B., Gates, S.N., Tse, E., Bart, S.M., Castellano, L.M., DiMaio, F., Shorter, J., and Southworth, D.R. (2019). Structural basis for substrate gripping and translocation by the ClpB $\mathrm{AAA}+$ disaggregase. Nat. Commun. 10, 1.

Rohou, A., and Grigorieff, N. (2015). CTFFIND4: Fast and accurate defocus estimation from electron micrographs. J. Struct. Biol. 192, 2, 216-221.

Román-Hernández, G., Grant, R.A., Sauer, R.T., and Baker, T.A. (2009). Molecular basis of substrate selection by the $\mathrm{N}$-end rule adaptor protein ClpS. Proc. Natl. Acad. Sci. U. S. A. 106, 22, 8888-8893.

Román-Hernández, G., Hou, J.Y., Grant, R.A., Sauer, R.T., and Baker, T.A. (2011). The ClpS adaptor mediates staged delivery of $\mathrm{N}$-end rule substrates to the AAA+ CIpAP protease. Mol. Cell 43, 2, 217-228.

Sandate, C.R., Szyk, A., Zehr, E.A., Lander, G.C., and Roll-Mecak, A. (2019). An allosteric network in spastin couples multiple activities required for microtubule severing. Nat. Struct. Mol. Biol. 26, 8, 671-678.

Sauer, R.T., and Baker, T.A. (2011). AAA+ proteases: ATP-fueled machines of protein destruction. Annu. Rev. Biochem. 80, 1, 587-612.

Schlieker, C., Weibezahn, J., Patzelt, H., Tessarz, P., Strub, C., Zeth, K., Erbse, A., SchneiderMergener, J., Chin, J.W., Schultz, P.G., et al. (2004). Substrate recognition by the AAA+ chaperone ClpB. Nat. Struct. Mol. Biol. 11, 7, 607-615.

Seol, J.H., Yoo, S.J., Kang, M.-S., Ha, D.B., and Chung, C.H. (1995). The 65-kDa protein derived from the internal translational start site of the clpA gene blocks autodegradation of ClpA by the ATP-dependent protease Ti in Escherichia coli. FEBS Lett. 377, 1, 41-43.

Sharipo, A., Imreh, M., Leonchiks, A., Brändén, C.I., and Masucci, M.G. (2001). cis-Inhibition of proteasomal degradation by viral repeats: Impact of length and amino acid composition. FEBS Lett. 499, 1-2, 137-142.

Shin, M., Watson, E.R., Song, A.S., Mindrebo, J.T., Novick, S.J., Griffin, P.R., Wiseman, R.L., and Lander, G.C. (2021). Structures of the human LONP1 protease reveal regulatory steps involved in protease activation. Nat. Commun. 12, 1, 3239. 
Singh, S.K., Grimaud, R., Hoskins, J.R., Wickner, S., and Maurizi, M.R. (2000). Unfolding and internalization of proteins by the ATP-dependent proteases CIpXP and ClpAP. Proc. Natl. Acad. Sci. U. S. A. 97, 16, 8898-8903.

Thompson, M.W., Singh, S.K., and Maurizi, M.R. (1994). Processive degradation of proteins by the ATP-dependent Clp protease from Escherichia coli. Requirement for the multiple array of active sites in ClpP but not ATP hydrolysis. J. Biol. Chem. 269, 27, 18209-18215.

Tobias, J.W., Shrader, T.E., Rocap, G., and Varshavsky, A. (1991). The N-end rule in bacteria. Science. 254, 5036, 1374-1377.

Too, P.H.M., Erales, J., Simen, J.D., Marjanovic, A., and Coffino, P. (2013). Slippery substrates impair function of a bacterial protease ATPase by unbalancing translocation versus exit. J. Biol. Chem. 288, 19, 13243-13257.

Torres-Delgado, A., Kotamarthi, H.C., Sauer, R.T., and Baker, T.A. (2020). The intrinsically disordered N-terminal extension of the ClpS adaptor reprograms its partner AAA+CIpAP protease. J. Mol. Biol. 432, 17, 4908-4921.

Twomey, E.C., Ji, Z., Wales, T.E., Bodnar, N.O., Ficarro, S.B., Marto, J.A., Engen, J.R., and Rapoport, T.A. (2019). Substrate processing by the Cdc48 ATPase complex is initiated by ubiquitin unfolding. Science. 365, 6452.

Varshavsky, A. (2019). N-degron and C-degron pathways of protein degradation. Proc. Natl. Acad. Sci. U. S. A. 116, 2, 358-366.

Vass, R.H., and Chien, P. (2013). Critical clamp loader processing by an essential AAA+ protease in Caulobacter crescentus. Proc. Natl. Acad. Sci. U. S. A. 110, 45, 18138-18143.

Wang, F., Mei, Z., Qi, Y., Yan, C., Hu, Q., Wang, J., and Shi, Y. (2011). Structure and mechanism of the hexameric MecA-ClpC molecular machine. Nature 471, 7338, 331-335.

Wang, K.H., Sauer, R.T., and Baker, T.A. (2007). ClpS modulates but is not essential for bacterial $\mathrm{N}$-end rule degradation. Genes Dev. 21, 4, 403-408.

Wang, K.H., Roman-Hernandez, G., Grant, R.A., Sauer, R.T., and Baker, T.A. (2008a). The molecular basis of $\mathrm{N}$-end rule recognition. Mol. Cell 32, 3, 406-414.

Wang, K.H., Oakes, E.S.C., Sauer, R.T., and Baker, T.A. (2008b). Tuning the strength of a bacterial $\mathrm{N}$-end rule degradation signal. J. Biol. Chem. 283, 36, 24600-24607.

Waterhouse, A.M., Procter, J.B., Martin, D.M.A., Clamp, M., and Barton, G.J. (2009). Jalview Version 2--a multiple sequence alignment editor and analysis workbench. Bioinformatics 25, 9, $1189-1191$.

Weibezahn, J., Tessarz, P., Schlieker, C., Zahn, R., Maglica, Z., Lee, S., Zentgraf, H., WeberBan, E.U., Dougan, D.A., Tsai, F.T.F., et al. (2004). Thermotolerance requires refolding of aggregated proteins by substrate translocation through the central pore of ClpB. Cell 119, 5, 653665.

White, K.I., Zhao, M., Choi, U.B., Pfuetzner, R.A., and Brunger, A.T. (2018). Structural principles of SNARE complex recognition by the AAA+ protein NSF. eLife 7, e38888.

Williams, C.J., Headd, J.J., Moriarty, N.W., Prisant, M.G., Videau, L.L., Deis, L.N., Verma, V., Keedy, D.A., Hintze, B.J., Chen, V.B., et al. (2018). MolProbity: More and better reference data for improved all-atom structure validation. Protein Sci. 27, 1, 293-315. 
Yokom, A.L., Gates, S.N., Jackrel, M.E., Mack, K.L., Su, M., Shorter, J., and Southworth, D.R. (2016). Spiral architecture of the Hsp104 disaggregase reveals the basis for polypeptide translocation. Nat. Struct. Mol. Biol. 23, 9, 830-837.

Yu, H., Lupoli, T.J., Kovach, A., Meng, X., Zhao, G., Nathan, C.F., and Li, H. (2018). ATP hydrolysis-coupled peptide translocation mechanism of Mycobacterium tuberculosis ClpB. Proc. Natl. Acad. Sci. U. S. A. 115, 41, E9560-E9569.

Zehr, E.A., Szyk, A., Szczesna, E., and Roll-Mecak, A. (2020). Katanin grips the $\beta$-tubulin tail through an electropositive double spiral to sever microtubules. Dev. Cell 52, 1, 118-131.e6.

Zeth, K., Dougan, D.A., Cusack, S., Bukau, B., and Ravelli, R.B. (2002a). Crystallization and preliminary X-ray analysis of the Escherichia coli adaptor protein ClpS, free and in complex with the N-terminal domain of ClpA. Acta Crystallogr. Sect. D Biol. Crystallogr. 58, 7, 1207-1210.

Zeth, K., Ravelli, R.B., Paal, K., Cusack, S., Bukau, B., and Dougan, D.A. (2002b). Structural analysis of the adaptor protein ClpS in complex with the N-terminal domain of ClpA. Nat. Struct. Biol. 9, 12, 906-911.

Zhao, J., Yu, X., Zhu, M., Kang, H., Ma, J., Wu, M., Gan, J., Deng, X., and Liang, H. (2016). Structural and molecular mechanism of CdpR involved in quorum-sensing and bacterial virulence in Pseudomonas aeruginosa. PLoS Biol. 14, 4, e1002449.

Zheng, S.Q., Palovcak, E., Armache, J.-P., Verba, K.A., Cheng, Y., and Agard, D.A. (2017). MotionCor2: anisotropic correction of beam-induced motion for improved cryo-electron microscopy. Nat. Methods 14, 4, 331-332.

Zivanov, J., Nakane, T., Forsberg, B.O., Kimanius, D., Hagen, W.J., Lindahl, E., and Scheres, S.H. (2018). New tools for automated high-resolution cryo-EM structure determination in RELION3. eLife 7, e42166.

Zuromski, K.L., Sauer, R.T., and Baker, T.A. (2020). Modular and coordinated activity of AAA+ active sites in the double-ring ClpA unfoldase of the ClpAP protease. Proc. Natl. Acad. Sci. U. S. A. $117,41,25455-25463$.

Zuromski, K.L., Kim, S., Sauer, R.T., and Baker, T.A. (2021). Division of labor between the pore1 loops of the D1 and D2 AAA+ rings coordinates substrate selectivity of the ClpAP protease. J. Biol. Chem. 297, 6, 101407. 